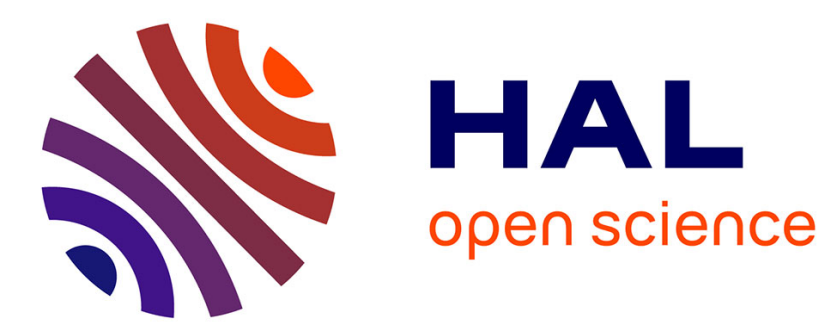

\title{
Guaranteed confidence region characterization for source localization using RSS measurements
}

\author{
Cheng-Yu Han, Michel Kieffer, Alain Lambert
}

\section{To cite this version:}

Cheng-Yu Han, Michel Kieffer, Alain Lambert. Guaranteed confidence region characterization for source localization using RSS measurements. Signal Processing, 2018, 10.1016/j.sigpro.2018.03.018 . hal-01754770

\section{HAL Id: hal-01754770}

https://hal-centralesupelec.archives-ouvertes.fr/hal-01754770

Submitted on 30 Mar 2018

HAL is a multi-disciplinary open access archive for the deposit and dissemination of scientific research documents, whether they are published or not. The documents may come from teaching and research institutions in France or abroad, or from public or private research centers.
L'archive ouverte pluridisciplinaire HAL, est destinée au dépôt et à la diffusion de documents scientifiques de niveau recherche, publiés ou non, émanant des établissements d'enseignement et de recherche français ou étrangers, des laboratoires publics ou privés. 


\title{
Guaranteed confidence region characterization
}

\section{for source localization using RSS measurements}

\author{
Cheng-Yu Han ${ }^{a}$ Michel Kieffer ${ }^{b}$ Alain Lambert ${ }^{a}$ \\ ${ }^{a}$ LRI, Univ Paris-Sud, CNRS, CentraleSupelec, F-91190 Gif-sur-Yvette \\ (e-mail: firstname.lastname@lri.fr). \\ b L2S, Univ Paris-Sud, CNRS, CentraleSupelec, F-91190 Gif-sur-Yvette (e-mail: firstname.lastname@lss.supelec.fr)
}

\begin{abstract}
This paper considers source localization in a Wireless Sensor Network (WSN) from Received Signal Strength (RSS) measurements. Its aim is to characterize confidence regions (CR) of the search space to which the source parameters (location, reference power, path loss exponent) are guaranteed to belong with a pre-specified probability level. The Leave-out Signdominant Correlation Regions (LSCR) method is adapted to this source localization context. LSCR defines CR considering very mild assumptions on the measurement noise corrupting the RSS readings. The confidence level may be arbitrarily chosen and the LSCR approach is valid even when very few measurements are available (non-asymptotic regime). The CR, as defined by LSCR may be non-convex or even non-connected sets. Their characterization is performed via interval analysis, which provides inner and outer approximations of CR.
\end{abstract}

The CR obtained via LSCR are compared to set estimates obtained using (robust) bounded-error estimation techniques, as well as to more classical confidence ellipsoids derived from Cramér-Rao lower bounds.

Key words: Source localization, confidence region characterization, RSS measurements, interval analysis

\section{Introduction}

Wireless sensor networks (WSNs) play an important role in applications such as environmental monitoring, source tracking, etc. In WSNs, the locations of sensing nodes are often required to make the collected information useful. Node localization algorithms have thus been developed, usually relying on a number of anchor nodes, whose location 
is known. Localization is mainly performed considering the received signal strength (RSS), the time of arrival (TOA), the time difference of arrival (TDOA) of electromagnetic or acoustic signals transmitted by a node to the anchor nodes or by the anchor nodes to the node to localize, see $(8 ; 20 ; 11)$.

In this paper, one considers guaranteed confidence region characterization for the location estimate of nodes using RSS measurements. In most of the papers, location estimates are evaluated first, and approximate characterization of confidence regions are then provided. Here, one evaluates first accurate estimates of confidence regions with prescribed confidence levels. Estimates may then be obtained using classical techniques, or may be evaluated as the barycenter of the confidence region. For that purpose, the Leave-out Sign-dominant Correlated Regions (LSCR) (1) approach is employed to define a region in the parameter space guaranteed to contain the true value of the parameter with an arbitrary confidence level. Confidence regions as defined by LSCR are valid even in the non-asymptotic regime, i.e., when only a small set of measurements is available.

In most of the papers, the RSS measurement noise is assumed to be log-normal or normal, see $(9 ; 34)$. This allows one to apply classical least-squares (LS), maximum likelihood (ML), or maximum a posteriori (MAP) estimation techniques. The main difficulty is that the cost function associated to the LS, ML, or MAP estimation problem may be non-convex, especially when the path-loss exponent as well as the reference transmission power have to be estimated jointly with the location. The differential RSS measurement localization approach proposed in (36) avoids estimating the reference power and facilitates estimation, e.g., via the total least-squares approach considered in (30). Convex $(32)$ or semi-definite $(17 ; 18 ; 35),(28 ; 34)$ relaxation techniques have been proposed, to facilitate the optimization. Using a semi-definite relaxation, (21) presents a minimax approach involving again the differential RSS measurements. In most of these approaches, the estimator confidence region is then defined using the Cramér-Rao lower bound (CRLB) (33). Nevertheless, confidence regions obtained from CRLB are usually optimistic and provide a relatively inaccurate confidence region when there are few measurements. Alternative bounded-error estimation techniques have been proposed in $(24 ; 19 ; 25)$ to get guaranteed set estimates. In these latter approaches, the RSS measurement errors are assumed to be bounded with known bounds. One tries to evaluate the set of all source locations that are consistent with the measurement model and the noise bounds. The resulting set is guaranteed to contain the true location of the source node, provided that the hypotheses on the noise bounds are satisfied. Nevertheless, in practice, noise samples do not really follow a normal or log-normal distribution, and the bounds considered in bounded-error estimation are either often violated because they are too small and result in an empty set estimate, or lead to huge solution sets when the bounds are too large. 
LSCR belongs to a family of recently-proposed techniques to define non-asymptotic confidence regions (NACR) $(1 ; 2 ; 3 ; 5 ; 6 ; 37)$. The main assumptions of LSCR are $(i)$ that the system output corresponds to the model output for a given true value of the parameter vector $(i i)$ that the measurement noise samples are independent and symmetrically distributed. Considering a given confidence level, and a finite set of measurements, LSCR defines a subset of the parameter space that is guaranteed to contains the true value of the parameter vector with a probability equal to the confidence level. Considering relatively mild conditions on the measurement noise, one is thus able to obtain guaranteed confidence regions. Nevertheless, the confidence region as defined by LSCR may be non-convex or even may consist of several disconnected components. As shown in (16), using tools from interval analysis (12), one is able to obtain inner and outer approximations of the confidence regions as defined by LSCR using subpavings, i.e., set of non-overlapping interval vectors.

The aim of this paper is to show that LSCR can be put at work to characterize NACR in the context of source localization from RSS measurements. Section 2 recalls the node localization problem from RSS measurements. The LSCR approach is then presented in Section 3, and the way this technique may be applied to the characterization of confidence regions for the node localization problem is detailed. Section 4 recalls some alternative approaches for the confidence region characterizations. In Section 5, the behavior of various tools from interval analysis to improve the characterization of NACR as defined by LSCR is analyzed. The performance of the proposed approach is also compared to the various approaches described in Section 4. Finally, Section 6 provides some conclusions and future research directions.

\section{Problem Formulation and Assumptions}

Consider a set of $n_{\mathrm{a}}$ anchor nodes situated on a plane, with fixed and known locations $\boldsymbol{\theta}_{i}, i=1, \ldots, n_{\mathrm{a}}$, in some reference frame $\mathcal{R}$ attached to the plane. This paper aims at providing a guaranteed NACR for the unknown location $\boldsymbol{\theta}_{0}$ in $\mathcal{R}$ of some static node, i.e., for a given confidence level, to provide a subset of locations to which the node belongs with the considered confidence level. For that purpose, the node periodically emits some electromagnetic or acoustic signal, which is received by the anchors. The $k$-th RSS measurement performed by anchor node $i$ is denoted $y(i, k)$. The anchor nodes transmit the RSS measurements to some central processing unit in charge of estimating $\boldsymbol{\theta}_{0}$ and the associated confidence region from $y(i, k), i=1, \ldots, n_{\mathrm{a}}, k=1, \ldots, n$. 
We assume that the $k$-th measurement obtained by anchor node $i$ is described by the Okumura-Hata model (27)

$$
y(i, k)=P_{0}-10 \gamma_{\mathrm{P}} \log _{10} \frac{\left\|\boldsymbol{\theta}_{0}-\boldsymbol{\theta}_{i}\right\|}{d_{0}}+w(i, k),
$$

where $P_{0}$ is the signal power measured at some known reference distance $d_{0}$ (typically $1 \mathrm{~m}$ ), $\gamma_{\mathrm{P}}$ is the path-loss exponent, $\|\cdot\|$ is the Euclidian norm, and $w(i, k)$ is the measurement noise. One assumes that $\gamma_{\mathrm{P}}$ is the same for all anchors, which is realistic in free-space propagation. Usually, the $w(i, k) \mathrm{s}$ are assumed to be realizations of some Gaussian random variables. In this paper, one only assumes that the $w(i, k) \mathrm{s}$ are realizations of independently, not necessarily identically distributed random variables with distributions symmetric around zero. This assumption is satisfied for Gaussian random variables when measurements are in the log domain, e.g., in dBm. Since $P_{0}$ and $\gamma_{\mathrm{P}}$ are not necessarily known by the anchors nodes, contrary to $d_{0}$, one considers the parameter vector $\mathbf{p}=\left[\boldsymbol{\theta}_{0}^{T}, P_{0}, \gamma_{\mathrm{P}}\right]^{T}$ for which a confidence region has to be estimated and from which a confidence region for $\boldsymbol{\theta}_{0}$ may then be deduced.

Assuming that there exists some true value $\mathbf{p}^{*}=\left[\boldsymbol{\theta}_{0}^{* T}, P_{0}^{*}, \gamma_{\mathrm{P}}^{*}\right]^{T}$ of the parameter vector, (1) can be rewritten as

$$
y(i, k)=y^{\mathrm{m}}\left(i, \mathbf{p}^{*}\right)+w(i, k)
$$

with

$$
y^{\mathrm{m}}\left(i, \mathbf{p}^{*}\right)=P_{0}^{*}-10 \gamma_{\mathrm{P}}^{*} \log _{10} \frac{\left\|\boldsymbol{\theta}_{0}^{*}-\boldsymbol{\theta}_{i}\right\|}{d_{0}}
$$

One assumes further that the search for the confidence region for $\mathbf{p}^{*}$ has to be done in some known compact set $\mathbb{P}_{0}$, which may be chosen arbitrarily large.

\section{Proposed Approach}

LSCR $(1 ; 2 ; 7)$ defines a set $\mathbb{P} \subset \mathbb{P}_{0}$ that can be proved to contain $\mathbf{p}^{*}$ with a prescribed probability. The main idea of LSCR is to consider various estimates of the correlations (as in (1)) or of higher-order statistics (as in (7)) of the prediction residuals, i.e., the differences between the measurements and model outputs for some value $\mathbf{p} \in \mathbb{P}_{0}$. Then regions of $\mathbb{P}_{0}$ are eliminated when too many estimates have the same sign, which contradicts the hypothesis that the noise samples are independent and symmetrically distributed. The regions that were not eliminated constitute the non-asymptotic confidence region $\mathbb{P}$. 


\subsection{Confidence Regions as Defined by LSCR}

First consider the vectors

$$
\begin{gathered}
\mathbf{y}(i)=(y(i, 1), \ldots, y(i, n))^{T} \\
\mathbf{y}_{\mathrm{m}}(i, \mathbf{p})=\left(y^{\mathrm{m}}(i, \mathbf{p}), \ldots, y^{\mathrm{m}}(i, \mathbf{p})\right)^{T}
\end{gathered}
$$

gathering the RSS measurements and the model output for the $i$-th anchor node. Then, consider the vectors

$$
\begin{aligned}
\mathbf{y} & =\left(\mathbf{y}^{T}(1), \ldots, \mathbf{y}^{T}\left(n_{\mathrm{a}}\right)\right)^{T} \\
\mathbf{y}_{\mathrm{m}}(\mathbf{p}) & =\left(\mathbf{y}_{\mathrm{m}}^{T}(1, \mathbf{p}), \ldots, \mathbf{y}_{\mathrm{m}}^{T}\left(n_{\mathrm{a}}, \mathbf{p}\right)\right)^{T}
\end{aligned}
$$

both with $n_{\mathrm{y}}=n_{\mathrm{a}} \times n$ entries, gathering in $n_{\mathrm{a}} \times n$ measurements and $n_{\mathrm{a}} \times n$ model outputs. Considering some value $\mathbf{p}$ of the parameter vector, one may introduce the prediction residual

$$
\mathbf{w}(\mathbf{p})=\mathbf{y}-\mathbf{y}_{\mathrm{m}}(\mathbf{p})
$$

When $\mathbf{p}=\mathbf{p}^{*}, \mathbf{w}\left(\mathbf{p}^{*}\right)$ contains the $n_{\mathrm{a}} \times n$ realizations of the measurement noise for all anchors at all measurement time instants.

To define a NACR, LSCR introduces three integers $s \geqslant 0, m>0$, and $q>0$, and a vector $\mathbf{e}=\left(e_{0}, \ldots, e_{s}\right)^{T}$ of non-negative integers such that at least one $e_{j}, 0 \leqslant j \leqslant s$ is odd. Considering some $\mathbf{p} \in \mathbb{P}_{0}$, for $t=1, \ldots, n_{\mathrm{y}}-s$, one can evaluate

$$
c_{t, \mathbf{e}}(\mathbf{p})=\prod_{i=0}^{s} w_{t+i}^{e_{i}}(\mathbf{p})
$$

where $w_{t}(\mathbf{p})$ is the $t$-th component of $\mathbf{w}(\mathbf{p})$. Then, using (9), one may compute several estimates of the higher-order statistic $E\left(\prod_{i=0}^{s} w_{t+i}^{e_{i}}(\mathbf{p})\right)$ of the prediction residuals, for $j=1, \ldots, m$

$$
s_{j, \mathbf{e}}(\mathbf{p})=\frac{1}{\left|\mathbb{I}_{j}^{n_{\mathrm{y}}-s}\right|} \sum_{\ell \in \mathbb{I}_{j}^{n_{\mathrm{y}}-s}} c_{\ell, \mathbf{e}}(\mathbf{p})
$$

where $\mathbb{I}_{j}^{n_{\mathrm{y}}-s}$ is a subset of the set $\mathbb{I}^{n_{\mathrm{y}}-s}=\left\{1, \ldots, n_{\mathrm{y}}-s\right\}$ of indexes such that the collection $\mathbb{G}=\left\{\mathbb{I}_{1}^{n_{\mathrm{y}}-s}, \ldots, \mathbb{I}_{m}^{n_{\mathrm{y}}-s}\right\}$ of these subsets forms a group under symmetric difference, i.e., $\forall \mathbb{I}_{j} \in \mathbb{G}^{n_{\mathrm{y}}-s}$ and $\forall \mathbb{I}_{j^{\prime}} \in \mathbb{G}^{n_{\mathrm{y}}-s}$, one has $\left(\mathbb{I}_{j} \cup \mathbb{I}_{j^{\prime}}\right)-$ $\left(\mathbb{I}_{j} \cap \mathbb{I}_{j^{\prime}}\right) \in \mathbb{G}^{n_{\mathrm{y}}-s}$. A procedure to build such $\mathbb{G}^{n_{\mathrm{y}}-s}$ is described in (7). In (10), $\left|\mathbb{I}_{j}^{n_{\mathrm{y}}-s}\right|$ is the cardinal number of $\mathbb{I}_{j}^{n_{\mathrm{y}}-s}$. 
Then, the set

$$
\mathbb{P}_{\mathbf{e}, q, m}=\left\{\mathbf{p} \in \mathbb{P}_{0} \mid \sum_{i=1}^{m} \tau_{i, \mathbf{e}}^{-}(\mathbf{p}) \geqslant q \text { and } \sum_{i=1}^{m} \tau_{i, \mathbf{e}}^{+}(\mathbf{p}) \geqslant q\right\}
$$

where

and

$$
\tau_{i, \mathbf{e}}^{-}(\mathbf{p})= \begin{cases}0 & \text { if } s_{i, \mathbf{e}}(\mathbf{p})<0 \\ B_{0.5} & \text { if } s_{i, \mathbf{e}}(\mathbf{p})=0 \\ 1 & \text { if } s_{i, \mathbf{e}}(\mathbf{p}) \dot{\iota} 0\end{cases}
$$

has been shown in $(1 ; 7)$ to be such that

$$
\tau_{i, \mathbf{e}}^{+}(\mathbf{p})= \begin{cases}0 & \text { if } s_{i, \mathbf{e}}(\mathbf{p})>0 \\ B_{0.5} & \text { if } s_{i, \mathbf{e}}(\mathbf{p})=0 \\ 1 & \text { if } s_{i, \mathbf{e}}(\mathbf{p})<0\end{cases}
$$

$$
\operatorname{Pr}\left(\mathbf{p}^{*} \in \mathbb{P}_{\mathbf{e}, q, m}\right)=1-\frac{2 q}{m}
$$

In (12) and (13), $B_{0.5}$ is a Bernoulli random variable with $\operatorname{Pr}\left(B_{0.5}=0\right)=\operatorname{Pr}\left(B_{0.5}=1\right)=0.5$ to solve ties.

The set $\mathbb{P}_{\mathbf{e}, q, m}$ contains all values of $\mathbf{p} \in \mathbb{P}_{0}$ such that at least $q$ out of the $m s_{i, \mathbf{e}}(\mathbf{p})$ are positive and $q$ are negative. It is a NACR containing $\mathbf{p}^{*}$ with a probability that is exactly $1-\frac{2 q}{m}$.

As indicated in (7), the choice of e depends on the considered estimation problem. It is discussed in Section 5.2 .

\subsection{Characterizing Confidence Regions}

The characterization of confidence regions as defined by LSCR is performed in (1) by sampling points in $\mathbb{P}_{0}$ and determining whether they belong to $\mathbb{P}_{\mathbf{e}, q, m}$. One thus gets an inner approximation of $\mathbb{P}_{\mathbf{e}, q, m}$, consisting of a union of points in $\mathbb{P}_{0}$ which have been proved to belong to $\mathbb{P}_{\mathbf{e}, q, m}$. When the confidence region is small, consists of several disconnected subsets, and when the number of sampling points is not enough, there is a risk to be unable to find any point in $\mathbb{P}_{\mathbf{e}, q, m}$, except with a fine gridding of $\mathbb{P}_{0}$.

An alternative approach based on interval analysis has been proposed in (16). Its main property is to yield inner and outer approximations of $\mathbb{P}_{\mathbf{e}, q, m}$ consisting of unions of non-overlapping boxes. This approach is briefly recalled in what follows. 


\subsubsection{Interval analysis}

An interval $[x]=[\underline{x}, \bar{x}]$ is a connected subset of $\mathbb{R}$. Interval analysis extends classical arithmetic operations and function evaluations to intervals.

Consider a function $\mathbf{f}$ from $\mathcal{D} \subset \mathbb{R}^{n}$ to $\mathbb{R}^{m}$ and some box (vector of intervals) $[\boldsymbol{x}] \subset \mathcal{D}$. Then the range of $\mathbf{f}$ over $[\boldsymbol{x}]$ is

$$
\mathbf{f}([\boldsymbol{x}])=\{\mathbf{f}(\boldsymbol{x}) \mid \boldsymbol{x} \in[\boldsymbol{x}]\} .
$$

Interval analysis introduces the notion of inclusion function that enables one to get an outer-approximating box for $\mathbf{f}([\boldsymbol{x}])$. More specifically, an inclusion function $[\mathbf{f}]$ of $\mathbf{f}$ is such that

$$
\forall[\boldsymbol{x}] \subset \mathcal{D}, \mathbf{f}([\boldsymbol{x}]) \subset[\mathbf{f}]([\boldsymbol{x}])
$$

Usually, $[\mathbf{f}]([\boldsymbol{x}]) \neq \mathbf{f}([\boldsymbol{x}])$, but an outer-approximation already provides a powerful tool to perform guaranteed characterization of confidence regions as defined by LSCR. The natural inclusion function is obtained replacing all real variables, arithmetical operations, and elementary function evaluations by their interval counterparts. More sophisticated inclusion functions such as centered forms are described, e.g., in (12).

\subsubsection{SIVIA}

Characterizing $\mathbb{P}_{\mathbf{e}, q, m}$ may be formulated as a set-inversion (14) problem:

$$
\mathbb{P}_{\mathbf{e}, q, m}=\left\{\mathbf{p} \in \mathbb{P}_{0} \mid \mathbf{f}_{\mathbf{e}, m}(\mathbf{p}) \in \mathbb{Y}_{q}\right\}=\mathbb{P}_{0} \cap \mathbf{f}_{\mathbf{e}, m}^{-1}\left(\mathbb{Y}_{q}\right),
$$

where

and

$$
\mathbf{f}_{\mathbf{e}, m}(\mathbf{p})=\left[\begin{array}{c}
f_{\mathbf{e}, m}^{+}(\mathbf{p}) \\
f_{\mathbf{e}, m}^{-}(\mathbf{p})
\end{array}\right]=\left[\begin{array}{c}
\sum_{i=1}^{m} \tau_{i, \mathbf{e}}^{+}(\mathbf{p}) \\
\sum_{i=1}^{m} \tau_{i, \mathbf{e}}^{-}(\mathbf{p})
\end{array}\right],
$$

$$
\mathbb{Y}_{q}=\left[\begin{array}{c}
\mathbb{Y}_{q}^{+} \\
\mathbb{Y}_{q}^{-}
\end{array}\right]=\left[\begin{array}{c}
{[q, \infty[} \\
{[q, \infty[}
\end{array}\right],
$$

which may be efficiently solved via interval analysis $(12 ; 23)$ using the SIVIA algorithm (12). For that purpose, inclusion functions for the $\mathbf{f}_{\mathbf{e}, m}$ are required. SIVIA, see Algorithm 1 recursively partitions $\mathbb{P}_{0}$ into three different 
types of boxes: boxes proved to belong to $\mathbb{P}_{\mathbf{e}, q, m}$ (see Line 1 ), boxes proved to have no intersection with $\mathbb{P}_{\mathbf{e}, q, m}($ see Line 2), and small undetermined boxes for which no conclusion can be obtained (Line 3). Undetermined boxes [p] are bisected into two subboxes $L[\mathbf{p}]$ and $R[\mathbf{p}]$ on which SIVIA is applied again, until their width is less than some precision parameter $\varepsilon$.

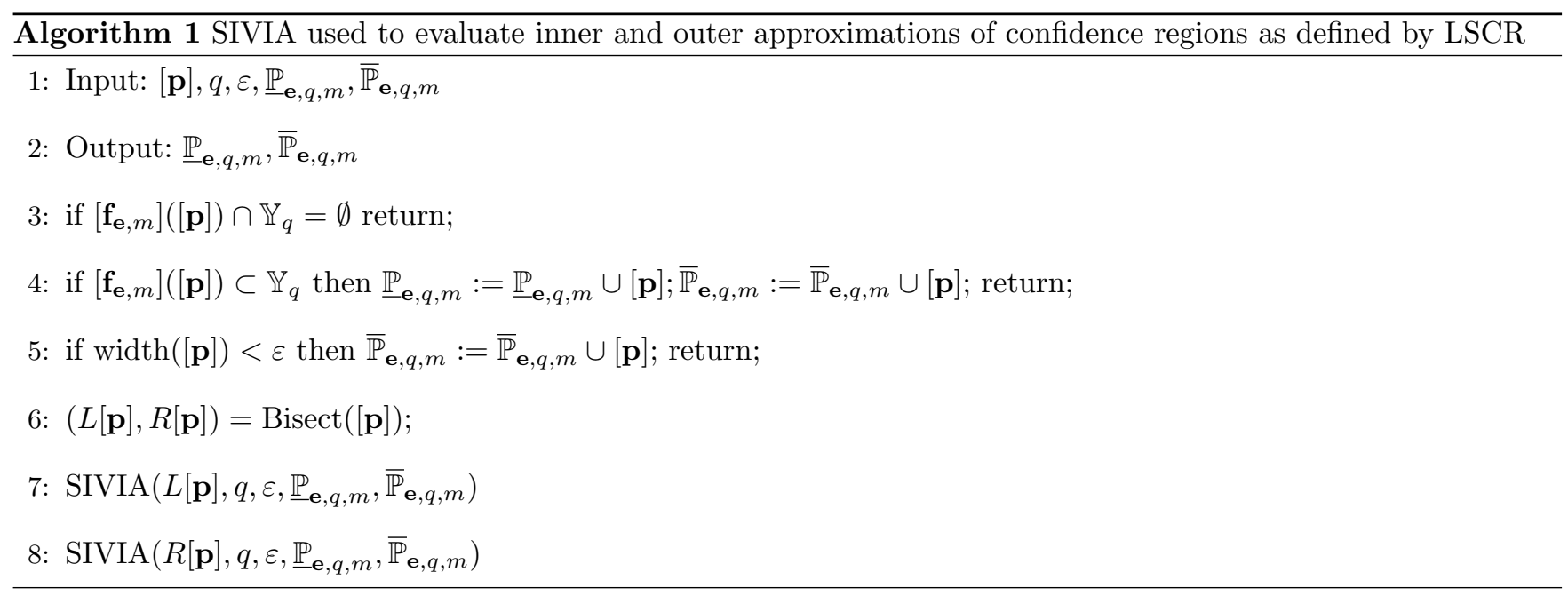

\subsubsection{Contractor}

Indetermination often results from range overestimation by inclusion functions. As a consequence, boxes have to be bisected by SIVIA many times to allow one to conclude on the position of the resulting boxes with respect to $\mathbb{P}_{\mathbf{e}, m, q}$. This may entail a significant computational complexity, even for a moderate dimension of p. Contractors may be useful in this context.

One may rewrite (17) as follows

$$
\begin{gathered}
\mathbb{P}_{\mathbf{e}, q, m}=\mathbb{P}^{+} \cap \mathbb{P}^{-} \\
\mathbb{P}^{+}=\left\{\mathbf{p} \in \mathbb{P}_{0} \mid f_{\mathbf{e}, m}^{+}(\mathbf{p}) \in \mathbb{Y}_{q}^{+}\right\}=\mathbb{P}_{0} \cap\left(f_{\mathbf{e}, m}^{+}\right)^{-1}\left(\mathbb{Y}_{q}^{+}\right) \\
\mathbb{P}^{-}=\left\{\mathbf{p} \in \mathbb{P}_{0} \mid f_{\mathbf{e}, m}^{-}(\mathbf{p}) \in \mathbb{Y}_{q}^{-}\right\}=\mathbb{P}_{0} \cap\left(f_{\mathbf{e}, m}^{-}\right)^{-1}\left(\mathbb{Y}_{q}^{-}\right) .
\end{gathered}
$$

A contractor $C_{f_{\mathbf{e}, m}^{+}, \mathbb{Y}_{q}^{+}}$associated, e.g., with the generic set-inversion problem (20) is a function taking a box $[\mathbf{p}]$ as input and returning a box $C_{f_{\mathbf{e}, m}^{+}, \mathbb{Y}_{q}^{+}}([\mathbf{p}]) \subset[\mathbf{p}]$ such that $[\mathbf{p}] \cap \mathbb{P}^{+}=C_{f_{\mathbf{e}, m}^{+}, \mathbb{Y}_{q}^{+}}([\mathbf{p}]) \cap \mathbb{P}^{+}$, so no part of $\mathbb{P}^{+}$in $[\mathbf{p}]$ is lost 
after contraction. Contractors eliminate parts of the candidate box $[\mathbf{p}]$ that do not belong to $\mathbb{P}^{+}$, without bisection.

Since $f_{\mathbf{e}, m}^{+}$and $f_{\mathbf{e}, m}^{-}$are not differentiable, most classical contractors, such as the forward-backward contractor, Newton's contractor, etc. (12), (4) cannot be used directly. To use contractors for the characterization of $\mathbb{P}^{+}$, considering a generic box $[\mathbf{p}]$, one first builds a set of $m$ subboxes of $[\mathbf{p}]$. The $i$-th subbox is obtained as the output of some contractor $C_{s_{i, \mathbf{e}},[0, \infty}\left[\right.$, trying to eliminate all $\mathbf{p} \in[\mathbf{p}]$ such that $s_{i, \mathbf{e}}(\mathbf{p})<0, i=1, \ldots, m$. Similarly, for $\mathbb{P}^{-}, m$ subboxes of $[\mathbf{p}]$ are built, each obtained as the output of some contractor $C_{\left.\left.s_{i, \mathbf{e}},\right]-\infty, 0\right]}$, trying to eliminate all $\mathbf{p} \in[\mathbf{p}]$ such that $s_{i, \mathbf{e}}(\mathbf{p})>0, i=1, \ldots, m$. Assuming that $\mathbf{y}_{\mathrm{m}}(\mathbf{p})$ is differentiable, the forward-backward contractor (12), the contractor based on the centred form (16), and the 3BCid contractor (4) may then be put at work. In a second step, the union of all intersections of $q$ out of the $m$ subboxes is evaluated and has been shown in (16) to provide a contractor $C_{f_{\mathbf{e}, m}^{+}, \mathbb{Y}_{q}^{+}}$associated with the generic set-inversion problem (20). A similar procedure may be considered for $C_{f_{e, m}^{-}, \mathbb{Y}_{q}^{-}}$associated with the generic set-inversion problem (21).

\section{Alternative approaches}

The aim of this section is to recall alternative approaches to which the NACR characterization will be compared in Section 5. On first starts with bounded-error (BE) and robust bounded-error (RBE) approaches.

\subsection{Bounded-Error Approach}

BE estimation techniques $(13 ; 22 ; 26)$ assume that the noise samples $w(i, k)$ in $(1)$ are bounded with known bounds, i.e., that $w(i, k) \in[\underline{w}(i, k), \bar{w}(i, k)]$, with $\underline{w}(i, k)$ and $\bar{w}(i, k)$ known for all $i=1 \ldots n_{a}$ and $k=1 \ldots n$. The set of all parameter vectors $\mathbf{p}$ belonging to some initial search set $\mathbb{P}_{0}$ that are consistent with the measurement model, the measurements, and the noise bounds is then characterized. This set may be defined as

$$
\mathbb{P}_{\mathrm{BE}}=\left\{\mathbf{p} \in \mathbb{P}_{0} \mid y_{\mathrm{m}}(i, \mathbf{p}) \in y(i, k)-[\underline{w}(i, k), \bar{w}(i, k)], i=1 \ldots n_{a}, k=1 \ldots n\right\} .
$$

Introducing $\overline{\mathbf{w}}(i)=(\bar{w}(i, 1), \ldots, \bar{w}(i, n))^{T}, \underline{\mathbf{w}}(i)=(\underline{w}(i, 1), \ldots, \underline{w}(i, n))^{T}, \overline{\mathbf{w}}=\left(\overline{\mathbf{w}}^{T}(1), \ldots, \overline{\mathbf{w}}^{T}\left(n_{\mathrm{a}}\right)\right)^{T}, \underline{\mathbf{w}}=$ $\left(\underline{\mathbf{w}}^{T}(1), \ldots, \underline{\mathbf{w}}^{T}\left(n_{\mathrm{a}}\right)\right)^{T}$ and

$$
[\mathbf{y}]=[\mathbf{y}-\overline{\mathbf{w}}, \mathbf{y}-\underline{\mathbf{w}}],
$$

one may formulate (22) as a set-inversion problem

$$
\mathbb{P}_{\mathrm{BE}}=\mathbb{P}_{0} \cap \mathbf{y}_{\mathrm{m}}^{-1}([\mathbf{y}]),
$$


which may be characterized using SIVIA.

The resulting set $\mathbb{P}_{\mathrm{BE}}$ is then guaranteed to contain the true value $\mathbf{p}^{*}$ of the parameter vector provided that all noise samples are within the considered bounds.

\subsection{Robust Bounded-Error Approach}

$\mathrm{BE}$ approaches may provide an empty set $\mathbb{P}_{\mathrm{BE}}=\emptyset$ as a result. Such result may be due to noise bounds which were violated by some measurements, to the initial search set which does not contain the solution set, or to the model which is a too coarse approximation of reality.

RBE estimation methods $(15 ; 29 ; 19)$ have been proposed to improve the robustness to outliers of BE estimation techniques. In (22), the solution set is defined as the set of parameter vectors that are consistent with all measurements. With RBE estimation, one tries to find the set of parameter vectors that are consistent with all but $\xi$ measurements and related noise bounds. In this context, the solution set may be formulated as

$$
\mathbb{P}_{\mathrm{RBE}, \xi}=\left\{\mathbf{p} \in \mathbb{P}_{0} \mid \tau(\mathbf{p}) \in \mathbb{Y}_{\xi}\right\},
$$

where

$$
\begin{gathered}
\tau(\mathbf{p})=\sum_{i=1}^{n_{a}} \sum_{k=1}^{n} \tau_{i, k}(\mathbf{p}), \\
\tau_{i, k}(\mathbf{p})= \begin{cases}1 & \text { if } y_{\mathrm{m}}(i, \mathbf{p}) \in y(i, k)-[\underline{w}(i, k), \bar{w}(i, k)], \\
0 & \text { else }\end{cases}
\end{gathered}
$$

and $\mathbb{Y}_{\xi}=\left[n_{a} n-\xi, n_{a} n\right]$.

Again, (22) can be viewed as a set-inversion problem

$$
\mathbb{P}_{\mathrm{RBE}, \xi}=\mathbb{P}_{0} \cap \tau^{-1}\left(\mathbb{Y}_{\xi}\right),
$$

which may be solved using SIVIA.

This approach does not need to specify in advance which measurements have to be considered as outliers. Considering a fixed value of $\xi$, one obtains an estimator robust against $\xi$ outliers. If $\xi$ is not fixed a priori, a possible strategy is to start with $\xi=0$, and to increase $\xi$ as long as an empty solution is provided by SIVIA. When a non-empty 
solution set has been obtained for some $\xi_{0} \geqslant 0$, one may consider $\xi=\xi_{0}+1$ or $\xi=\xi_{0}+2$ and evaluate $\mathbb{P}_{\mathrm{RBE}, \xi}$ to further increase the robustness of the estimated set $\mathbb{P}_{\mathrm{RBE}, \xi}$.

The resulting set $\mathbb{P}_{\mathrm{RBE}, \xi}$ is guaranteed to contain the true value $\mathbf{p}^{*}$ of the parameter vector provided that there are actually less than $\xi$ outliers.

\subsection{Nonlinear ML estimate and Cramér-Rao Lower Bound}

\subsubsection{Maximum Likelihood Estimation (MLE)}

Assuming that the noise samples $w(i, k)$ in (1) are independently and identically distributed (iid) as zero-mean Gaussian with variance $\sigma^{2}$, one may obtain the MLE of $\mathbf{p}^{*}$ as

$$
\begin{aligned}
\widehat{\mathbf{p}}_{\mathrm{MLE}} & =\arg \max _{\mathbf{p}} \pi(\mathbf{y} \mid \mathbf{p}) \\
& =\arg \min _{\mathbf{p}} \sum_{i=1}^{n_{\mathrm{a}}} \sum_{k=1}^{n}\left(y(i, k)-P_{0}+10 \gamma_{\mathrm{P}} \log _{10} \frac{\left\|\boldsymbol{\theta}_{0}-\boldsymbol{\theta}_{i}\right\|}{d_{0}}\right)^{2},
\end{aligned}
$$

where $\pi(\mathbf{y} \mid \mathbf{p})$ is the likelihood function, which requires a precise knowledge of the noise probability distribution. The cost function to minimize is usually non-convex and has several local minimizers. Nevertheless, one may try to minimize it using, e.g., lsqnonlin of Matlab.

\subsubsection{Cramér-Rao Lower Bound (CRLB)}

Once an estimate $\widehat{\mathbf{p}}_{\mathrm{MLE}}$ has found, the CRLB may be used to derive an ellipsoidal confidence region in the parameter space. Assuming that $\widehat{\mathbf{p}}_{\mathrm{MLE}}$ is unbiased, and introducing the estimator covariance matrix $\Sigma$, one has

$$
\Sigma \succcurlyeq \mathbf{J}^{-1}(\mathbf{p})
$$

where $\mathbf{J}(\mathbf{p})$ is the $\operatorname{dim}(\mathbf{p}) \times \operatorname{dim}(\mathbf{p})$ Fisher information matrix, with components

$$
J_{i_{1}, i_{2}}(\mathbf{p})=-E\left[\frac{\partial^{2} \ln \pi(\mathbf{y} \mid \mathbf{p})}{\partial p_{i_{1}} \partial p_{i_{2}}}\right]
$$

We have assumed that the noise samples were independent, thus (27) becomes

$$
J_{i_{1}, i_{2}}(\mathbf{p})=-\sum_{i=1}^{n_{a}} \sum_{k=1}^{n} E\left[\frac{\partial^{2} \ln \pi(y(i, k) \mid \mathbf{p})}{\partial p_{i_{1}} \partial p_{i_{2}}}\right]
$$


Using the approximation $\Sigma \simeq \mathbf{J}^{-1}(\mathbf{p})$, confidence ellipsoids with prescribed confidence levels may then be derived using $\mathbf{J}^{-1}(\mathbf{p})$, see (33).

\subsection{Semidefinite programming (SDP) with Cramér-Rao Lower Bound}

The approach considered in (34) consists in solving the localization problem via semidefinite programming. The confidence region is then evaluated considering confidence ellipsoid associated to the CRLB. This approach is briefly recalled here.

Only $\boldsymbol{\theta}_{0}$ and $P_{0}$ are assumed to be unknown. First, (1) is reformulated as

$$
\log _{10} d_{i}^{2} \lambda_{i}=\frac{P_{0}}{5 \gamma_{\mathrm{P}}}+\frac{w(i, k)}{5 \gamma_{\mathrm{P}}}
$$

where $d_{i}=\left\|\boldsymbol{\theta}_{0}-\boldsymbol{\theta}_{i}\right\|$ and $\lambda_{i}=10^{y(i, k) / 5 \gamma_{\mathrm{P}}}$. Introducing $\alpha=10^{P_{0} / 5 \gamma_{\mathrm{P}}}$, one deduces that

$$
d_{i}^{2} \lambda_{i}=\alpha 10^{w(i, k) / 5 \gamma_{\mathrm{P}}}
$$

Assuming that the noise is sufficiently small, the right-hand side of (30) is expanded as

$$
d_{i}^{2} \lambda_{i}=\alpha+\epsilon(i, k)
$$

where $\epsilon(i, k)=\frac{\ln 10}{5 \gamma_{\mathrm{P}}} w(i, k)$.

An estimator for $\mathbf{p}=\left(P_{0}, \boldsymbol{\theta}_{0}^{T}\right)^{T}$ is then expressed as

$$
\widehat{\mathbf{p}}=\arg \min _{P_{0}, \boldsymbol{\theta}_{0}} \sum_{i=1 \ldots n_{a}, k=1 \ldots n}\left(d_{i}^{2} \lambda_{i}-\alpha\right)^{2}
$$

Finding $\widehat{\mathbf{p}}$ is difficult in general, since the cost function is non-linear and non-convex. To address this issue, (34) 
introduce the auxiliary variable $z=\boldsymbol{\theta}_{0}^{T} \boldsymbol{\theta}_{0}$ to get the SDP problem

$$
\begin{gathered}
\left(\widehat{\alpha}, \widehat{z}, \widehat{\boldsymbol{\theta}}_{0}\right)=\min _{\alpha, z, \boldsymbol{\theta}_{0}} \sum_{i=1 \ldots n_{a}, k=1 \ldots n}\left(d_{i}^{2} \lambda_{i}-\alpha\right)^{2} \\
\text { subject to } d_{i}^{2}=\left[\begin{array}{c}
\boldsymbol{\theta}_{i} \\
-1
\end{array}\right]^{T}\left[\begin{array}{ll}
\mathrm{I}_{2} & \boldsymbol{\theta}_{0} \\
\boldsymbol{\theta}_{0}^{T} & z
\end{array}\right]\left[\begin{array}{l}
\boldsymbol{\theta}_{i} \\
-1
\end{array}\right], \\
{\left[\begin{array}{cc}
\mathrm{I}_{2} & \boldsymbol{\theta}_{0} \\
\boldsymbol{\theta}_{0}^{T} & z
\end{array}\right] \succeq 0 .}
\end{gathered}
$$

The solution of this problem may be obtained with standard SDP solvers such as SDPT3 (31). An estimate $\widehat{\mathbf{p}}_{\text {SDP }}$ is then easily deduced from $\widehat{\alpha}, \widehat{z}$, and $\widehat{\boldsymbol{\theta}}_{0}$. The confidence region in this case is again obtained from the CRLB.

\section{Simulation Results}

In this section, the LSCR technique, applied to source localization from anchor RSS measurements, is evaluated and compared to the BE and RBE estimation approaches as well as to the non-linear MLE and SDP-based estimation approach with confidence regions obtained from the CRLB.

\subsection{Simulation Setup}

The simulation setup considered here is the same as that in (34). Five anchor nodes $\left(n_{a}=5\right)$ are placed in the corners and in the center of a square of $20 \mathrm{~m} \times 20 \mathrm{~m}$; they are represented by black circles in the figures of Table 3 and 4. $N=32$ agents are regularly placed in the square; their location is indicated by crosses. At the beginning of the localization process, each agent broadcasts $n=10$ times a message containing its identifier. We assume that, using appropriate signaling, there is no collision between messages from different agents. Each anchor measures the RSS (in $\mathrm{dBm}$ ) of the messages as well as the agent identifier. These data are then transmitted to a central processing unit to determine an estimate of the position of the agents and its corresponding confidence region.

One has thus $n_{\mathrm{y}}=n \times n_{\mathrm{a}}=50$ measurements available for each agent. In the simulations, the reference power $P_{0}=30 \mathrm{dBm}$ at $d_{0}=1 \mathrm{~m}$ is the same for all agents. The path loss exponent is taken as $\gamma_{\mathrm{P}}=4$. Data are corrupted by two types of noise samples, assumed independent and identically distributed (iid). In a first set of experiments, the 
noise samples are realizations of iid zero-mean Gaussian random variables with standard deviation $\sigma_{0}=2 \mathrm{dBm}$. In a second set of experiments, the noise samples are realizations of iid Gaussian-Bernouilli-Gaussian (GBG) variables. In the latter case, with a probability $p_{0}=0.9$, the noise is zero-mean Gaussian with standard deviation $\sigma_{0}=2 \mathrm{dBm}$ and with a probability $p_{1}=0.1$, it is zero-mean Gaussian with standard deviation $\sigma_{1}=5 \mathrm{dBm}$.

Three estimation problems are considered:

(1) Only the location $\boldsymbol{\theta}_{0, i}, i=1, \ldots, N$ of each agent has to be estimated, $\gamma_{\mathrm{P}}$ and $P_{0}$ are assumed to be known. The search box for $\boldsymbol{\theta}_{0, i}$ is $[0,25] \times[0,25] \mathrm{m}^{2}$.

(2) $\boldsymbol{\theta}_{0, i}$ and $P_{0, i}, i=1, \ldots, N$ have to be determined for each agent, the unknown reference power is not assumed to be the same for all agents, contrary to $\gamma_{\mathrm{P}}$ which is assumed known. The search interval for the $P_{0, i}, i=1, \ldots, N$ is $[0 \mathrm{dBm}, 40 \mathrm{dBm}]$.

(3) $\boldsymbol{\theta}_{0, i}$ and $\gamma_{\mathrm{P}}, i=1, \ldots, N$ have to be determined for each agent, the reference power $P_{0, i}$ is assumed to be known for all agents (e.g., transmitted in the packet broadcast by each agent). The search interval for $\gamma_{\mathrm{P}}$ is $[2,8]$.

The goal is to get an estimate of the parameters in these three cases, as well as an estimate of the associated confidence region.

Note that the case of $\boldsymbol{\theta}_{0, i}, P_{0, i}, i=1, \ldots, N$ and $\gamma_{\mathrm{P}}$ unknown is not considered, since there is some identifiability issue in that case. In the model (3), the impact on the estimation of $\boldsymbol{\theta}_{0, i}$ of an uncertainty on $\gamma_{\mathrm{P}}$ or of $P_{0, i}$ is similar.

\subsection{Selection of the parameters of the LSCR approach}

To apply the proposed LSCR technique, several parameters have to be chosen. The most difficult to choose is e, which determines the considered statistic of the prediction residuals. Considering $s=1$ and $\mathbf{e}=(1,1)^{T}$ corresponds to the correlation function considered in (1), which provides satisfying results in the considered application, as will be seen in what follows.

Other choices are possible, but two aspects have to be taken into account. The first is related to the characterization by SIVIA of NACRs. In the inclusion function $\left[\mathbf{f}_{\mathbf{e}, m}\right]$ of $\mathbf{f}_{\mathbf{e}, m}$ used by SIVIA, all occurences of the parameters are considered as independent. The conservatism with which the range of $\mathbf{f}_{\mathbf{e}, m}$ is evaluated depends on the number of occurences of each parameter to be estimated in the formal expression of $\mathbf{f}_{\mathbf{e}, m}$, see (12). Thus, statistics with large values of $\sum_{i=0}^{s} e_{i}$ should be avoided to improve the efficiency of SIVIA. The second is related to the LSCR method 
itself: the correlation functions should lead to small and regularly-shaped confidence regions.

Some preliminary experiments have been conducted to evaluate the impact of e. One has considered $s=0$ and $s=1$ as well as $q=3$, and $m=63$. To build $\mathbb{G}$ used in $(1 ; 7)$, Gordon's procedure (10) is used, but each $\mathbb{I}_{j} \in \mathbb{G}$ consists only of the $n_{\mathrm{y}}-s$ elements provided by the original procedure. The choice $m=63$ allows an exploitation of all data, and avoids having duplicate elements in $\mathbb{G}$. As a consequence, $(m-2 q) / m \simeq 90 \%$ confidence regions are provided by LSCR.

Table 1 represents the NACR for the location $\boldsymbol{\theta}_{0}$ of an agent represented by a cross for different statistics (9) used by LSCR and defined by the vector e considering the organization of the measurement vector introduced in (6). The shape of the confidence regions is satisfying only when $\mathbf{e}=(1,1)^{T}$ or when $\mathbf{e}=(3,1)^{T}$. The sets in blue and red represent respectively the projections on the $\left(\theta_{1}, \theta_{2}\right)$-plane of the inner (blue boxes) and outer (blue and red boxes) approximations, respectively of $90 \%$ NACRs.

One may consider the following alternative organization of the measurement vector and apply the NACR characterization technique

$$
\begin{aligned}
\mathbf{y}(j) & =\left(y(1, j), \ldots, y\left(n_{\mathrm{a}}, j\right)\right)^{T} \\
\mathbf{y} & =\left(\mathbf{y}^{T}(1), \ldots, \mathbf{y}^{T}(n)\right)^{T} .
\end{aligned}
$$

But in that case, the shapes of the confidence regions are satisfying for none of the vectors $\mathbf{e}$.

To interpret the previous results, one has to consider the asymptotic analysis of the behavior of the LSCR approach provided in (7). A condition to get $\mathbb{P}_{\mathbf{e}, q, m}$ shrinking to $\mathbf{p}^{*}$ when $n_{\mathrm{y}}=n_{\mathrm{a}} \times n$ tends to infinity is that for any $\mathbf{p} \neq \mathbf{p}^{*}$, all $s_{j, \mathbf{e}}(\mathbf{p})$ should have the same sign as $n_{\mathrm{y}}$ tends to infinity. In Appendix A, one shows that this perperty is satisfied when considering $\mathbf{e}=(1,1)^{T}$ or $\mathbf{e}=(3,1)^{T}$ and an organization of the measurement vector as described in (6). One also shows that this is not possible considering an organization of the measurement vector as described in (36).

\subsection{Evaluation of the LSCR approach}

First, one evaluates NACR as defined by LSCR for $\mathbf{p}=\boldsymbol{\theta}_{0}$ only, using the plain SIVIA algorithm and compares its performance to a characterization via gridding, see Table 2 .

Both schemes provide sets of comparable shapes. When $\varepsilon$ decreases, the set of boxes provided by SIVIA and the 
$\mathbf{e}=(1)$

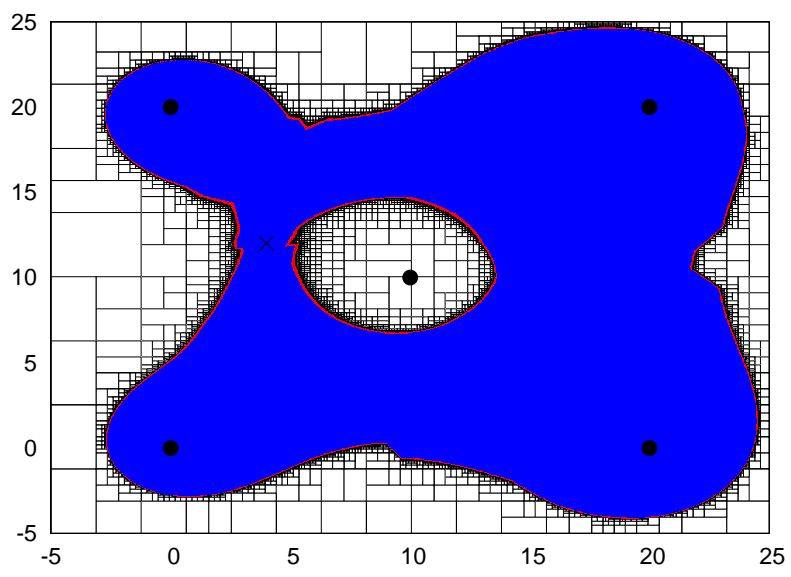

$\mathbf{e}=(1,1)^{T}$
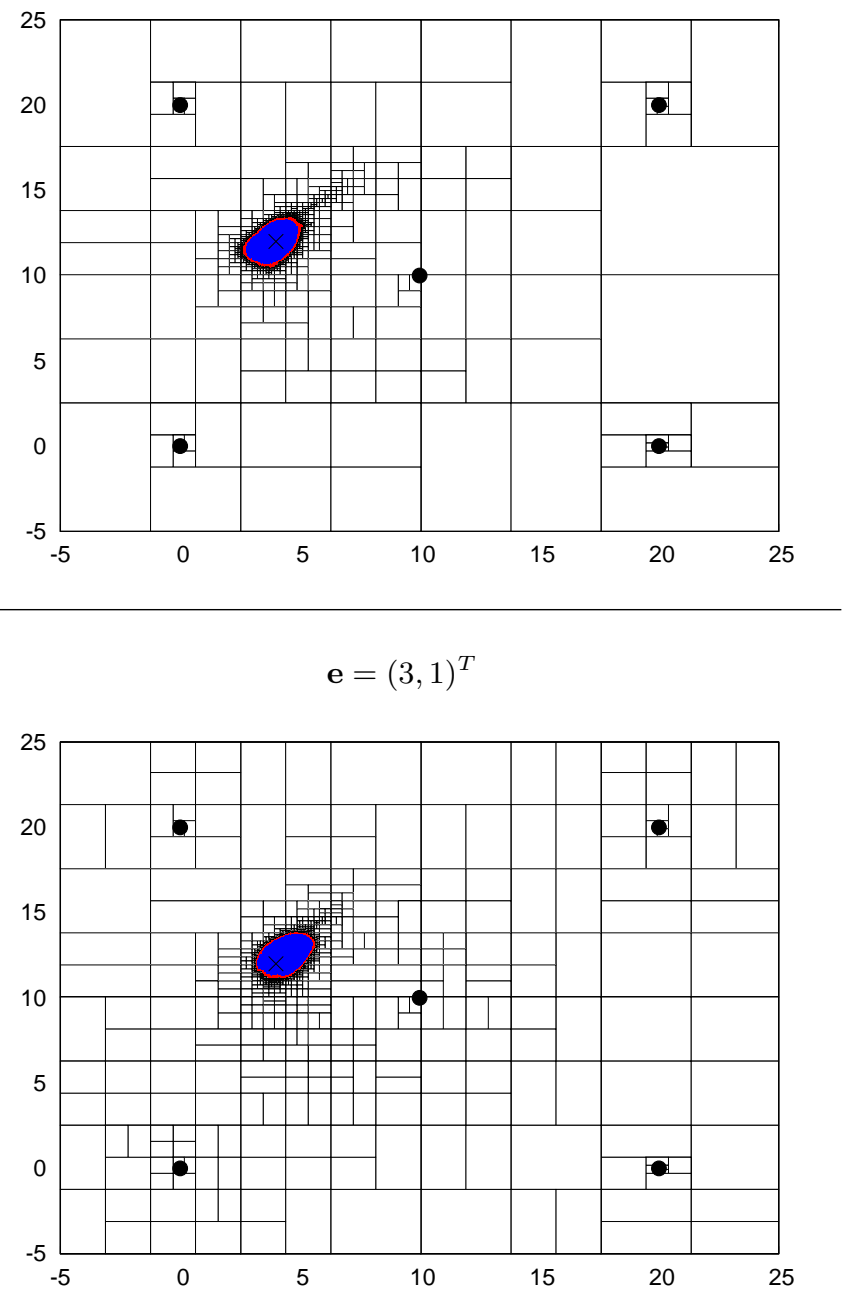

Table 1

NACR as defined by LSCR for the location of a given agent represented by a cross; the NACR are obtained for different statistics defined by e, with an organization of the measurement vector as described by (6).

set of points obtained by gridding become similar. Nevertheless, with $\varepsilon=0.2$ (resp. $\varepsilon=0.05$ ), characterization via SIVIA is 10 (resp. 100) times faster than via gridding. Gridding is faster than SIVIA only for large values of $\varepsilon$. This confirms results already obtained in (16).

Now, one aims at comparing SIVIA without contractors (NC) and variants of SIVIA involving the forward-backward contractor $(\mathrm{FB})$, the 3BCid contractor $(3 \mathrm{~B})$, and the contractor based on the centred form (CF). In all cases, these contractors are used to build, for some box $[\mathbf{p}]$, the $m$ subboxes of $[\mathbf{p}]$ such that $s_{i, r}^{e}(\mathbf{p}) \geqslant 0, i=1, \ldots, m$ and the $m$ subboxes of $[\mathbf{p}]$ such that $s_{i, r}^{\mathrm{e}}(\mathbf{p}) \leqslant 0, i=1, \ldots, m$, see $(12)$ and (13). The $q$-intersection contractor (16) is then 


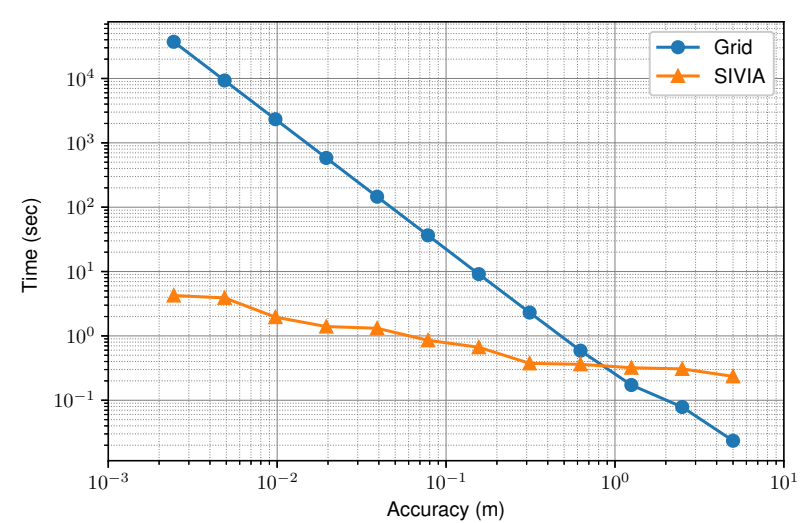

Table 2 (left) and set of points or subpavings (right) for $\varepsilon=0.2$ and $\varepsilon=0.05$.

applied on both subsets of $m$ boxes.

Figure 1 shows the size of the confidence region for $\mathbf{p}=\boldsymbol{\theta}_{0}$ when using the plain SIVIA, SIVIA with FB, 3B, and CF contractors. For each technique, two lines are provided. The upper lines represent the size of the outer approximation and the lower lines represent the size of the inner approximation of the NACR. When $\varepsilon$ is too large, no inner approximation can be obtained. The outer and inner approximation converge when the parameter $\varepsilon$ becomes smaller. For a given value of $\varepsilon$, the confidence region obtained with SIVIA and using contractors has a smaller size than without contractors. The 3B and CF contractors provide the best results.

Figure 2 shows the evolution of size of the outer and inner approximations of the NACR as a function of the time required to obtain it with the different variants of SIVIA without and with contractors. Again, when $\varepsilon$ is too large, no inner approximation can be obtained. Clearly, NC provides the best compromise between accuracy and computing time.

To understand the relative inefficiency of contractors in the context of NACR characterization, Figure 3 shows the contraction obtained on each box processed by SIVIA using the CF contractor. The x-axis represents the original surface of each processed box and the y-axis represents the surface of the box after contraction. One sees that large 


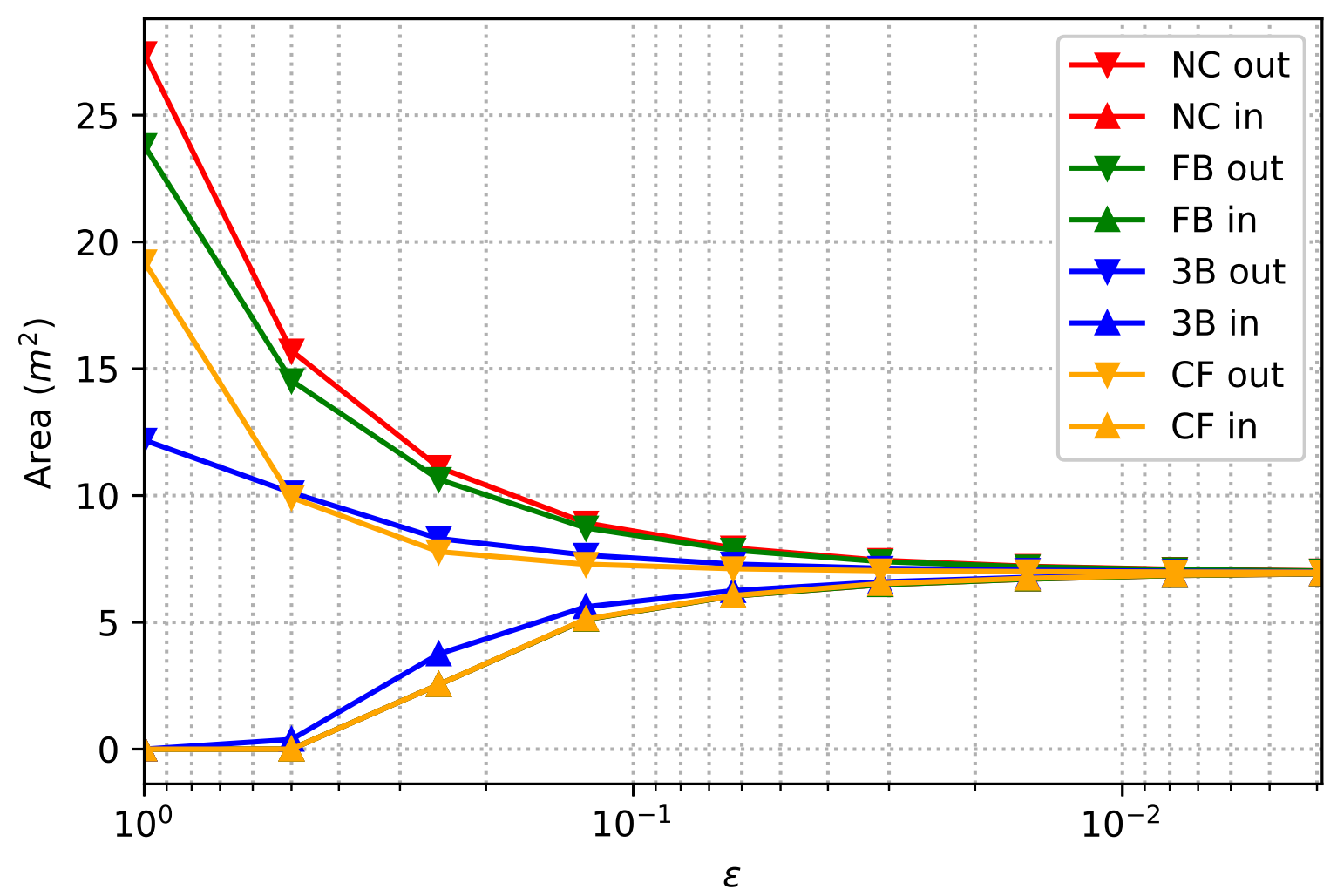

Fig. 1. Size of the outer (upper lines) and inner (lower lines) approximation of the confidence region as defined by LSCR provided by different variants of SIVIA, without and with contractors for different values of the precision parameter $\varepsilon$ boxes are left unchanged. The CF contractor is only able to contract relatively small boxes, which helps obtaining a more accurate description of the NACR, but with a significantly increased computational complexity. The computing time penalty to apply contractors is not compensated by the gain in terms of reduction of the size of the search space provided by contractors.

\subsection{Comparison with alternative methods}

Table 3 summarizes an example of estimation results obtained with the approaches described in Sections 3 and 4 considering Gaussian noise only. The columns correspond to different estimation problems. The rows correspond the results provided, respectively by LSCR, the BE, and the RBE estimators as well as the estimate obtained using a MLE approach with confidence regions evaluated from the CRLB approach presented in Section 4.3.

The intervals for the measurement noise are taken as $\left[-3 \sigma_{0}, 3 \sigma_{0}\right]$ for the BE approach and as $\left[-2 \sigma_{0}, 2 \sigma_{0}\right]$ for the 


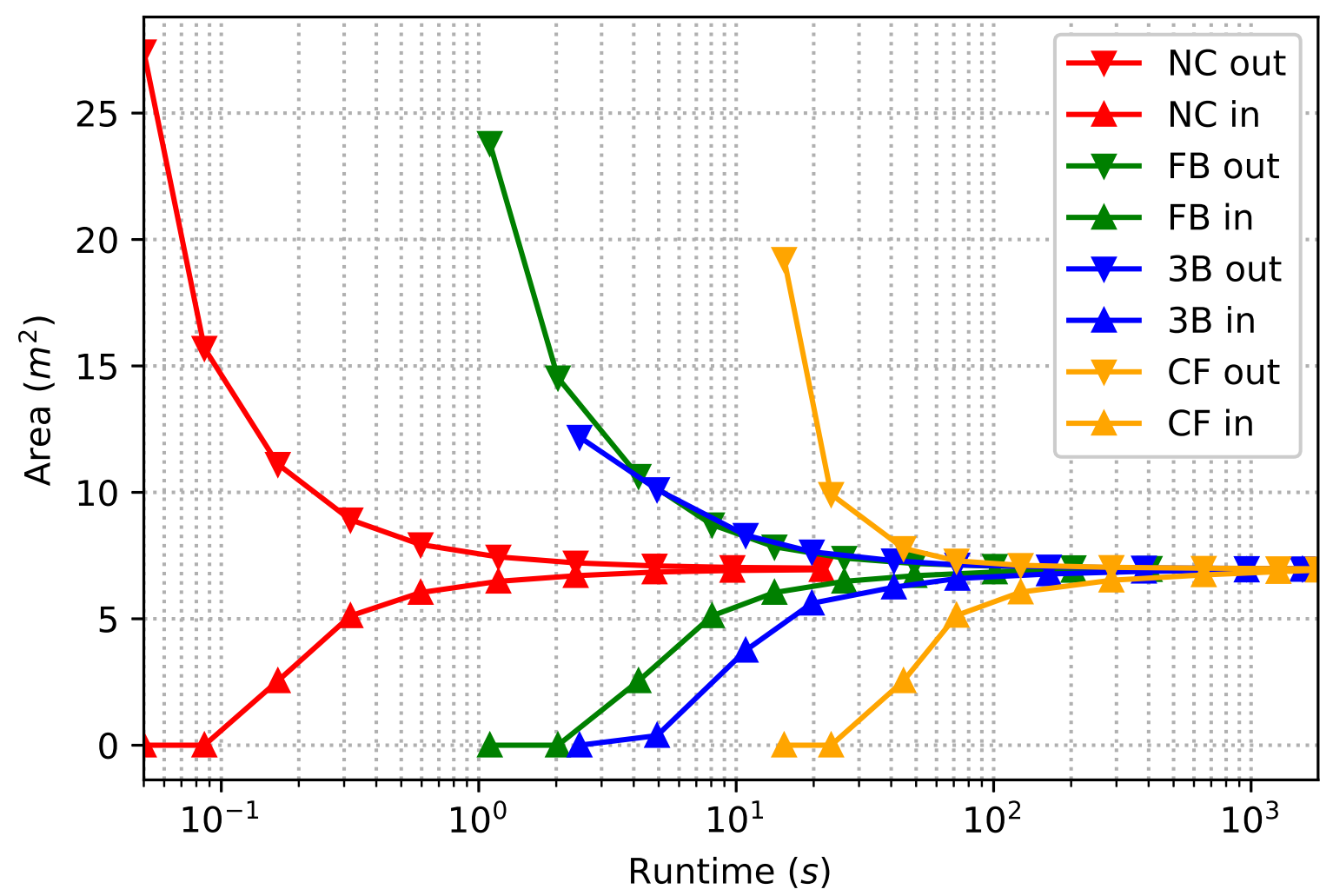

Fig. 2. Size of the outer (upper lines) and inner (lower lines) approximation of the confidence region as defined by LSCR provided by different variants of SIVIA, without and with contractors as a function of the computing time (the curves are parametrized in $\varepsilon$ )

RBE approach. The plain SIVIA algorithm without contractors is used with the LSCR, BE, and RBE approaches with $\varepsilon=0.05$. The sets in blue and red represent respectively the projections on the $\left(\theta_{1}, \theta_{2}\right)$-plane of the inner (blue boxes) and outer (blue and red boxes) approximations, respectively, of $90 \%$ NACRs (for LSCR), or of the set estimates (for the $\mathrm{BE}$ or RBE approaches). A triangle close to a node location indicate that the set provided for that node is either empty or does not contain the actual node location.

The initialization for the nonlinear MLE is taken at random within the convex hull of the anchors. Optimization is performed using Matlab lsqnonlin non-linear least-squares optimization function. With some initializations, Isqnonlin provides estimates which projection on the $\left(\theta_{1}, \theta_{2}\right)$-plane are outside the $[-5,25]^{2}$ box. In that case, a new search is performed with an other random initialization. This reinitialization is performed up to three times. The blue ellipses represent the $90 \%$ confidence region derived from the CRLB. The lines link the estimated positions 


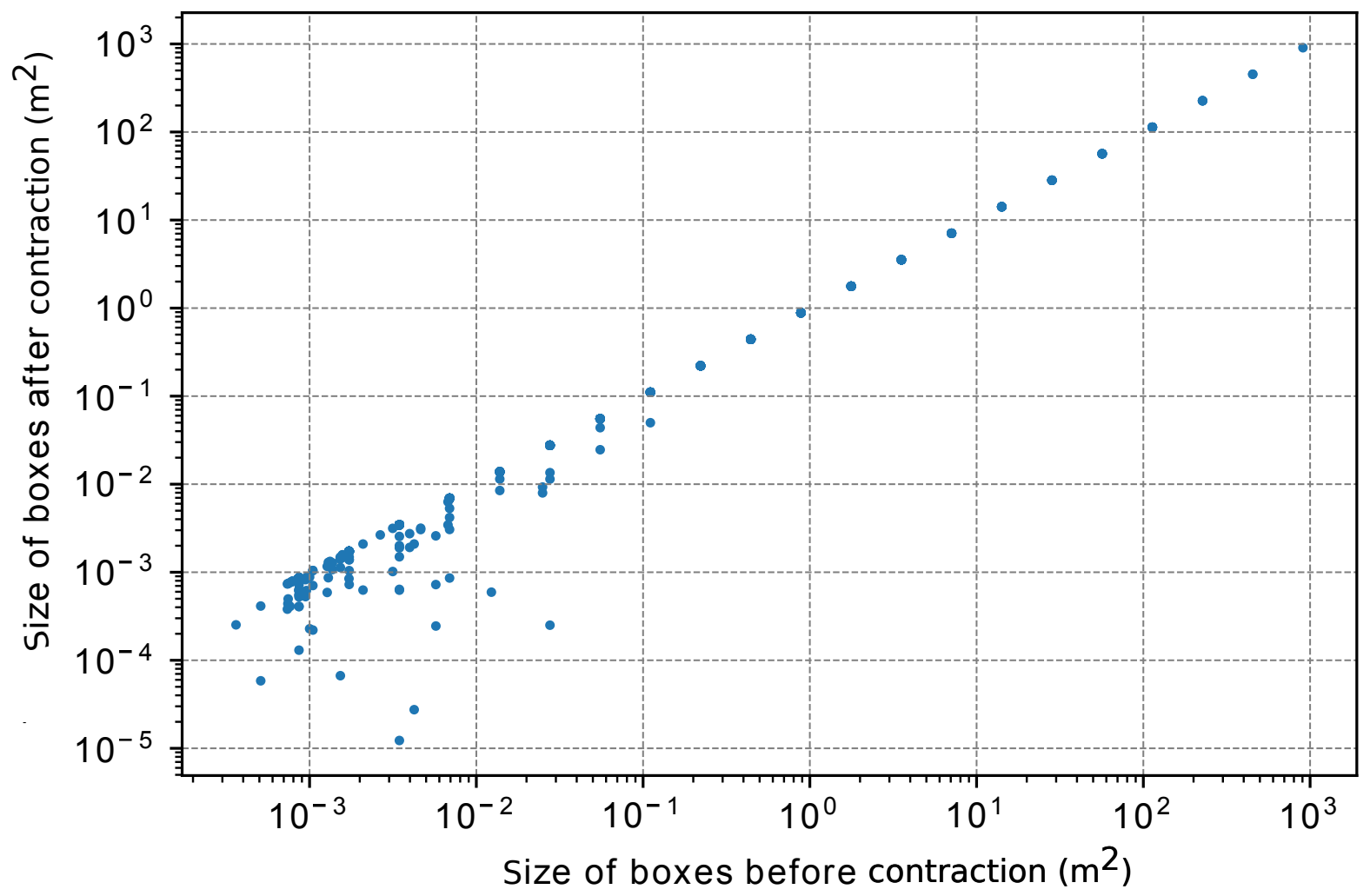

Fig. 3. Evolution of the surface of boxes before and after processing by the CF contractor $\hat{\theta}$ and true positions $\theta^{*}$ of the agents.

Table 4 is similar to Table 3 but with results obtained from measurements corrupted by GBG noise. The intervals for the measurement noise are again taken as $\left[-3 \sigma_{0}, 3 \sigma_{0}\right]$ for the BE approach and as $\left[-2 \sigma_{0}, 2 \sigma_{0}\right]$ for the RBE approach.

Table 5 provides the estimates of $\boldsymbol{\theta}_{0}$ obtained with the SDP approach assuming that both $\boldsymbol{\theta}_{0}$ and $P_{0}$ have to be estimated and the projection of the $90 \%$ confidence ellipsoid derived from the CRLB in the case of Gaussian noise only (left) and GBG noise (right).

Table 3 shows that when the number of the unknown variables increases, the size of the sets provided by the LSCR, $\mathrm{BE}$, and RBE approaches increases. This is due to the fact that an uncertainty in $P_{0}$ or $\gamma_{\mathrm{P}}$ translates directly in an uncertainty in terms of node localization. One also see that an uncertainty in $P_{0}$ or in $\gamma_{\mathrm{P}}$ have comparable impacts on the size and shape of the obtained sets. This is consistent with the identifiability issue mentionned in Section 5.1. Sometimes, the sets provided by LSCR are empty or do not contain the actual node location. This is consistent 
with the definition of a $90 \%$ confidence region, which should contain the actual location only in $90 \%$ of the cases. Considering GBG noise also increases the size of the sets obtained: Confidence regions in Table 4 tend to be larger then those in Table 3. This is not surprising, since data corrupted GBG noise contain less information about the node location than data corrupted by Gaussian noise

Nonlinear MLE provides good results when only $\boldsymbol{\theta}_{0}$ has to be estimated. Nevertheless, when both $\boldsymbol{\theta}_{0}$ and $P_{0}$ or $\boldsymbol{\theta}_{0}$ and $\gamma_{P}$ have to be estimated, the estimation errors increases significantly. SDP is more robust than nonlinear MLE when several parameters have to be estimated. In general, confidence regions derived from the CRLB are by far too optimistic: agents seldom belong to the $90 \%$ confidence ellipsoid.

In Table 3, the sets evaluated using BE are in general larger than those obtained with RBE or than the confidence regions defined by LSCR. In the case of GBG noise, most of the sets provided by the BE approach are empty. This is not surprising, since the assumption on the noise bounds is violated in many cases. The size of the sets evaluated with the RBE approach is not much affected by the nature of the noise, showing the robustness of the approach to variations of the noise characteristics. Nevertheless, in some cases, in presence of GBG noise, the sets provided by the RBE approach do not contain the true value of the location of some nodes. This is due to the fact that not enough outliers were tolerated.

\subsection{Effect of the noise variance}

In this section, one studies the effect of the noise variance on the size of the confidence region and on the location estimation error. The same simulation setup as in Section 5.1 is considered, but now, the $N=100$ agents to be localized are randomly placed in the square. Noise samples in (1) are realizations of iid zero-mean Gaussian random variables with constant standard deviation in each simulation. Experiments are performed with $\sigma_{0}$ ranging from 0.5 to $4 \mathrm{dBm}$. Both $\boldsymbol{\theta}_{0, i}$ and $P_{0, i}, i=1, \ldots, N$ have to be determined for each agent.

\subsubsection{Localization error}

To evaluate the localization error of the LSCR, BE, and RBE approaches, one considers the outer approximations $\overline{\mathbb{P}}_{\mathbf{e}, q, m}, \overline{\mathbb{P}}_{\mathrm{BE}}$, and $\overline{\mathbb{P}}_{\mathrm{RBE}}$ of $\mathbb{P}_{\mathbf{e}, q, m}, \mathbb{P}_{\mathrm{BE}}$, and $\mathbb{P}_{\mathrm{RBE}}$ and evaluate the center of mass of these sets defined, e.g., for $\overline{\mathbb{P}}_{\mathrm{BE}}$ as

$$
\widehat{\mathbf{p}}_{\mathrm{BE}}=\frac{1}{\operatorname{vol}\left(\overline{\mathbb{P}}_{\mathrm{BE}}\right)} \sum_{[\mathbf{p}] \in \overline{\mathbb{P}}_{\mathrm{BE}}} \operatorname{vol}([\mathbf{p}]) \operatorname{mid}([\mathbf{p}])
$$




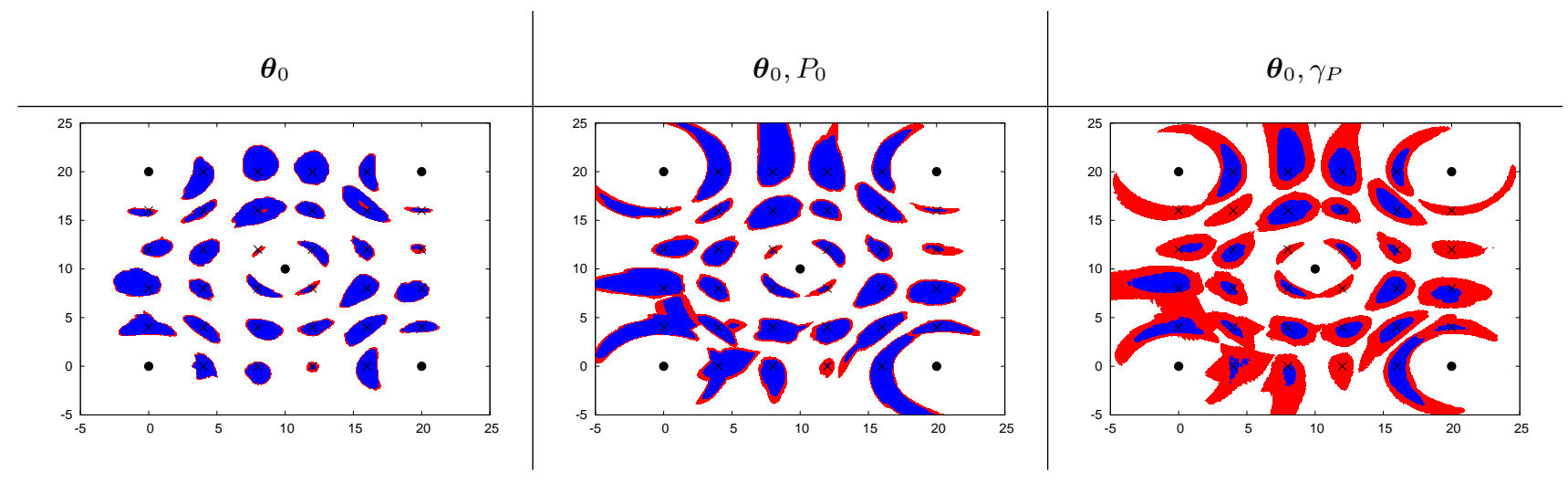

LSCR
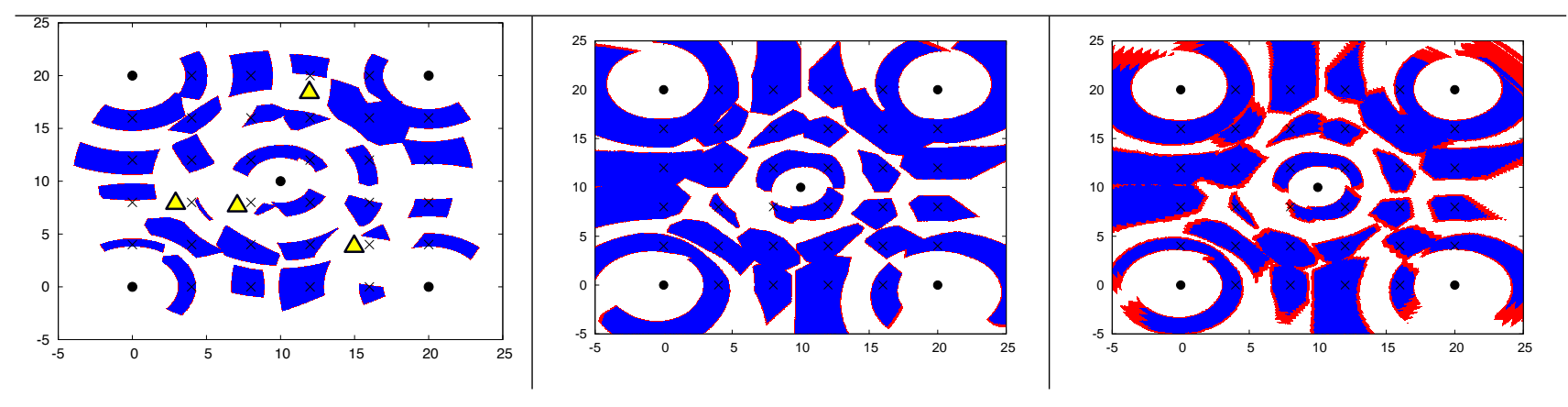

BE
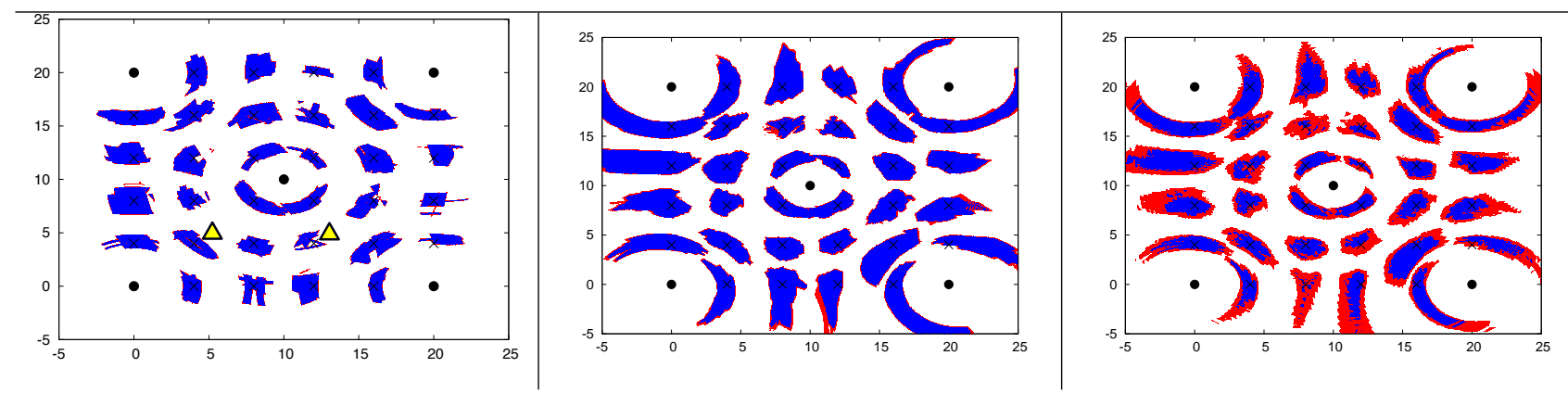

RBE
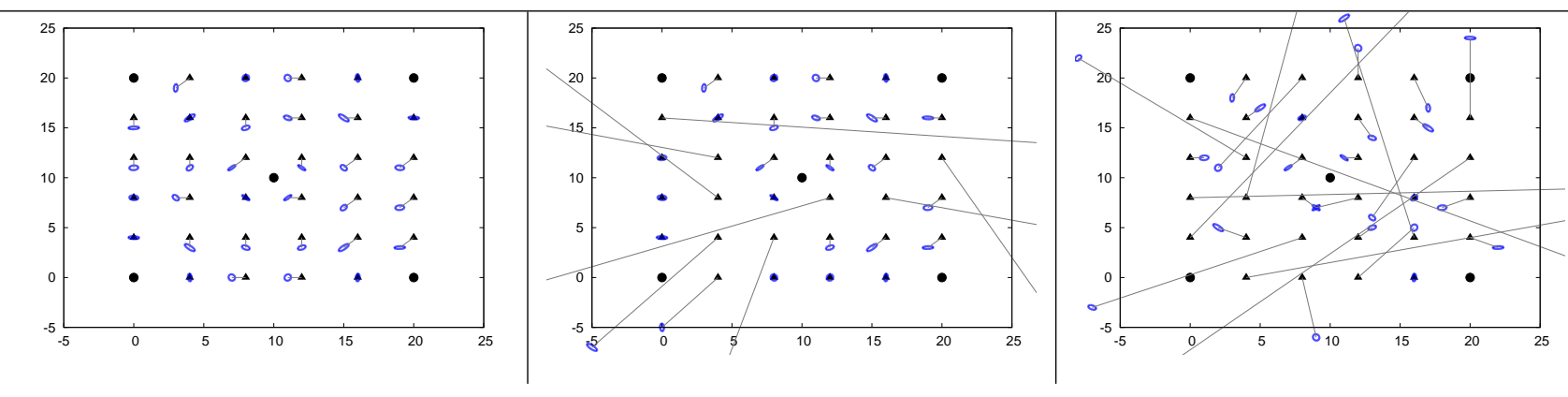

ML

Table 3

Simulations considering measurements corrupted by Gaussian noise: Projections on the $\left(\theta_{1}, \theta_{2}\right)$-plane of inner and outerapproximations of set estimates obtained by SIVIA in the case NACR as defined by LSCR, and in the case of Bounded-Error (BE), and Robust Bounded-Error (RBE) estimation, as well 22 MLE with confidence regions derived from the CRLB; nodes for which the set estimate is empty or does not contain its actual location are marked by a triangle. 


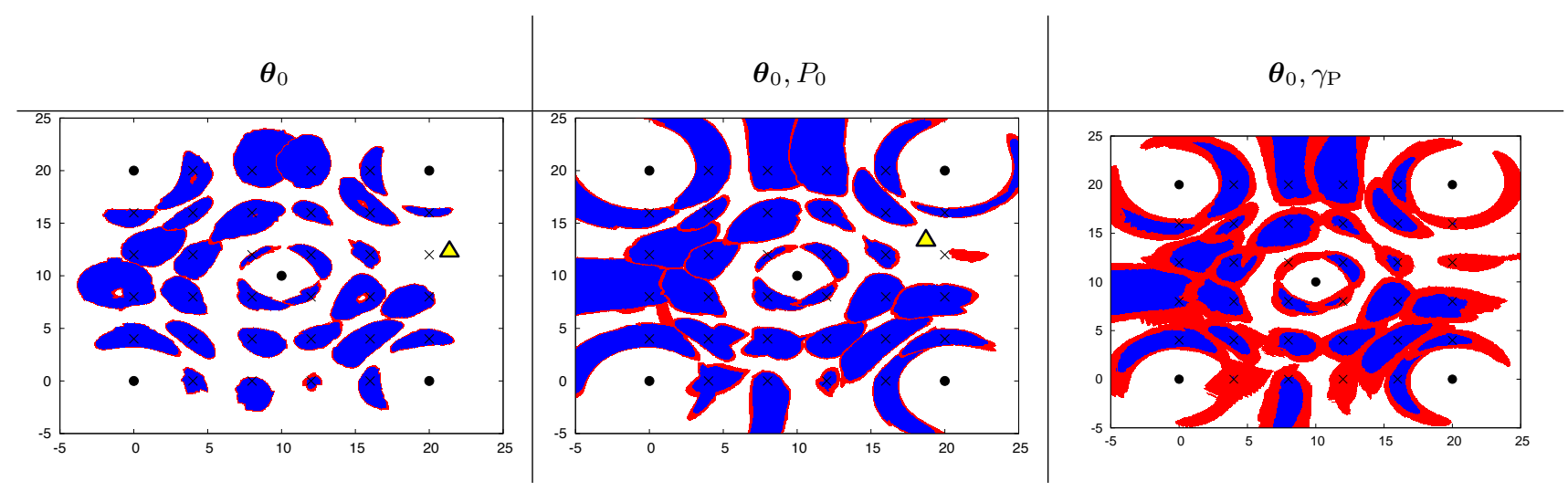

LSCR
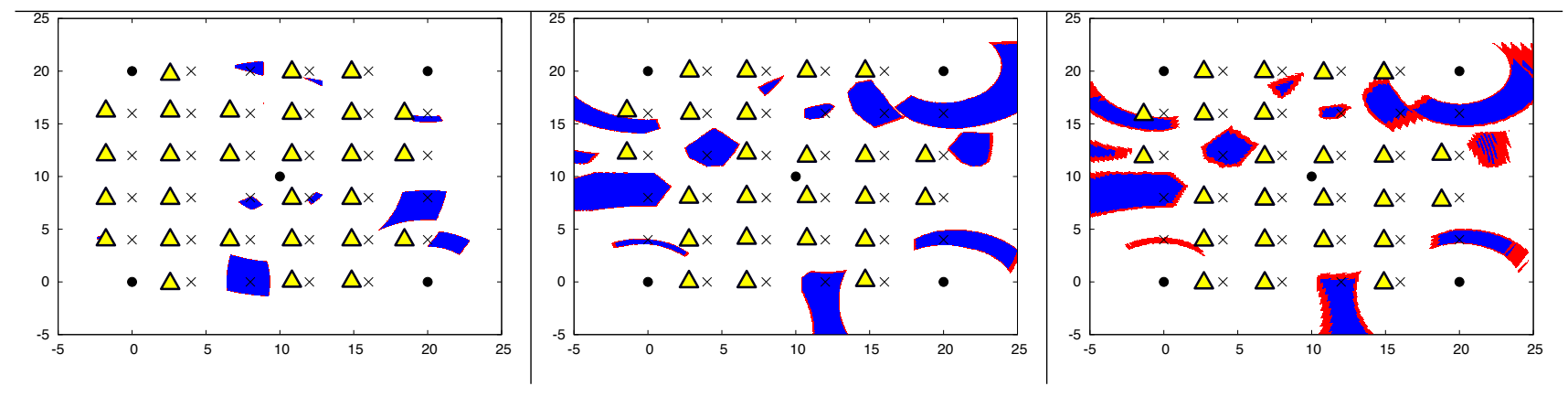

BE
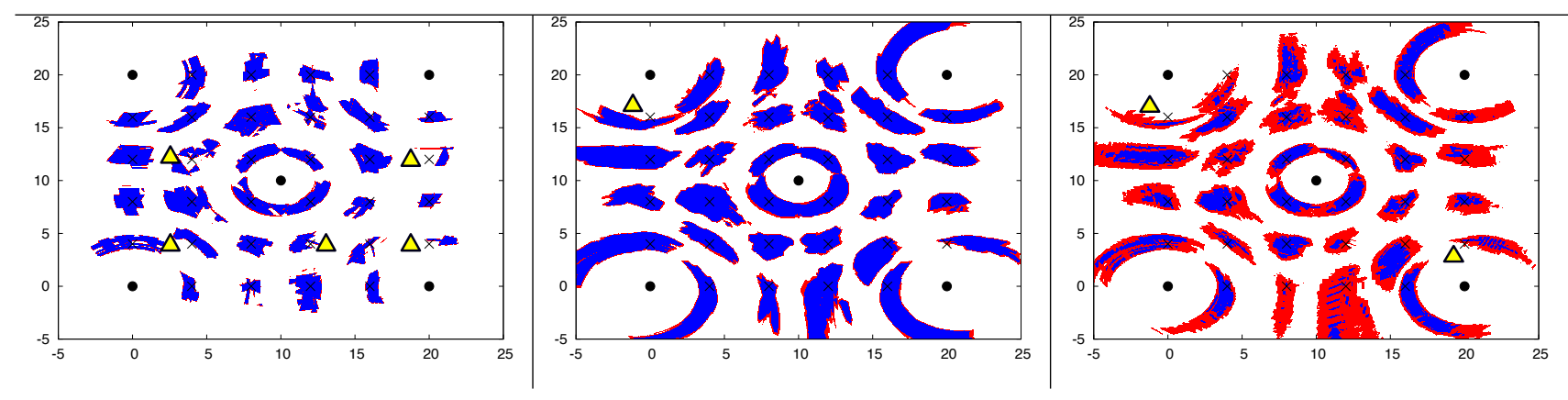

RBE
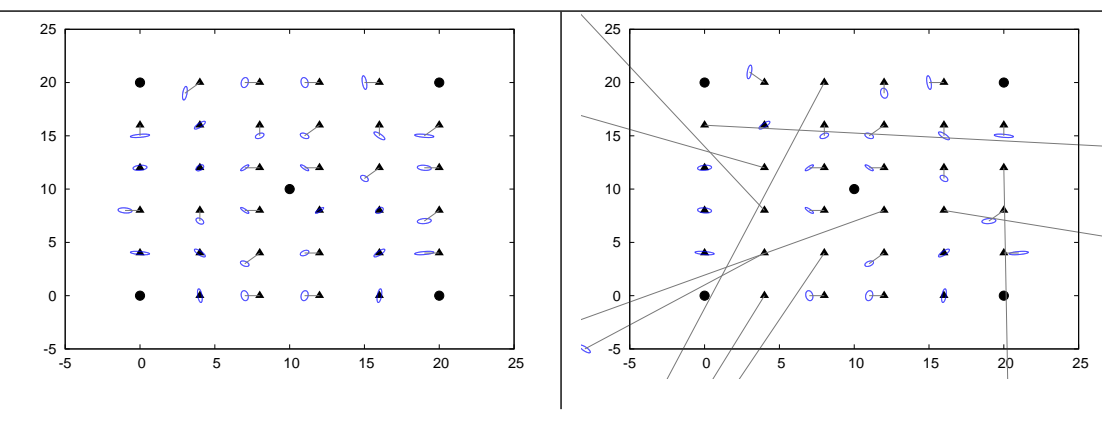

ML

Table 4

Simulations considering measurements corrupted by GBG noise: Projections on the $\left(\theta_{1}, \theta_{2}\right)$-plane of inner and outerapproximations of set estimates obtained by SIVIA in the case NACR as defined by LSCR, and in the case of Bounded-Error (BE), and Robust Bounded-Error (RBE) estimation, as well 23 MLE with confidence regions derived from the CRLB; nodes for which the set estimate is empty or does not contain its actual location are marked by a triangle. 

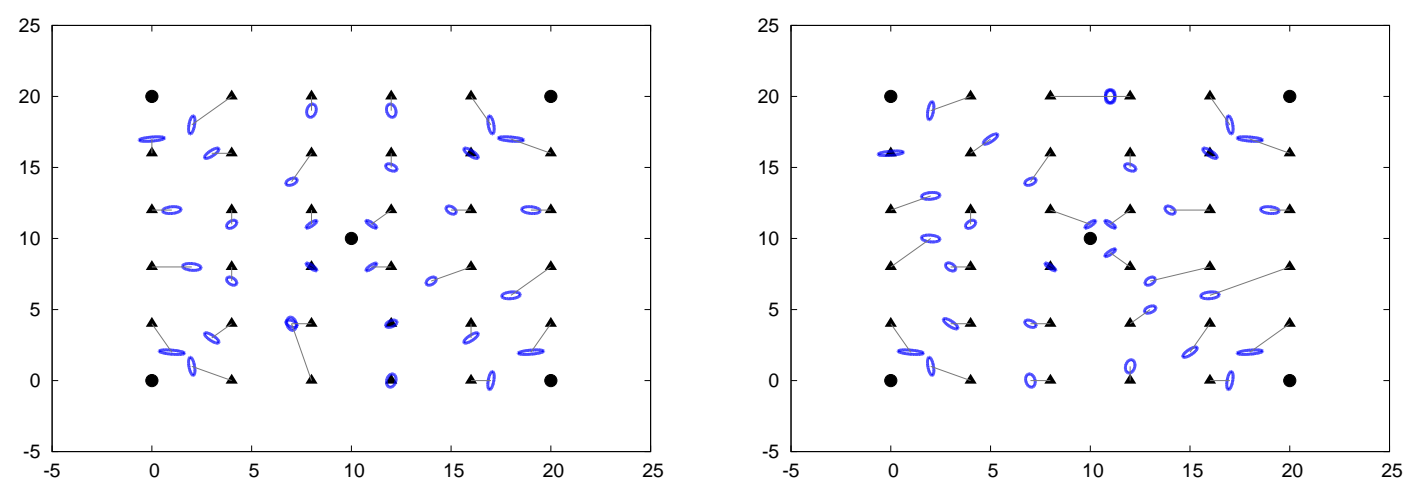

Table 5

Projection on the $\left(\theta_{1}, \theta_{2}\right)$-plane of estimates and confidence ellipsoids obtained by the SDP method in Gaussian noise (left) and GBG noise (right) in the case of

where $[\mathbf{p}]$ is a box contained in the list of non-overlapping boxes forming $\overline{\mathbb{P}}_{\mathrm{BE}}$, vol $([\mathbf{p}])$ and mid $([\mathbf{p}])$ are the volume and midpoint of $[\mathbf{p}]$. The estimated location $\widehat{\boldsymbol{\theta}}_{0, \mathrm{BE}}$ is the deduced from $\widehat{\mathbf{p}}_{\mathrm{BE}}$. When an empty set is provided by the LSCR or the BE estimation techniques, no point estimate is evaluated.

Figure 4 shows the root mean-square (RMS) error

$$
\delta=\sqrt{\frac{1}{N} \sum_{i=1}^{N}\left\|\widehat{\boldsymbol{\theta}}_{0, i}-\boldsymbol{\theta}_{0, i}\right\|^{2}}
$$

between the estimated location $\widehat{\boldsymbol{\theta}}_{0, i}$ and the actual location $\boldsymbol{\theta}_{0, i}$ as a function of the measurement noise standard deviation. For the LSCR and BE techniques, the average is only evaluated when the obtained sets are not empty.

For all estimation techniques, $\delta$ increases with $\sigma$. The LSCR and RBE methods provide the best results. The poor performance of SDP when $\sigma$ is small is due to the relaxation of the constraints. The poor performance of MLE is due to the fact that this approach fails to provide reasonable estimates for some of the sensors due to local minima of the cost function.

\subsubsection{Characteristics of the confidence region}

Figure 5 shows the proportion of agents actually located in the set estimates or in the $90 \%$ confidence region provided by the LSCR or derived from the CRLB. For BE, RBE, and LSCR, lower and upper bounds are provided, corresponding to the inclusion in the inner and outer approximation of the sets $\mathbb{P}_{\mathrm{BE}}, \mathbb{P}_{\mathrm{RBE}}$, and $\mathbb{P}_{\mathbf{e}, q, m}$, respectively. In SIVIA applied to characterize the confidence regions as defined by LSCR, $\varepsilon=10^{-2}$ if $\sigma_{0} \leqslant 1.5$ and $\varepsilon=5 \times 10^{-2}$ else. This explains the discontinuity in the curves for LSCR. Moreover, results have been averaged over 10 realizations. 


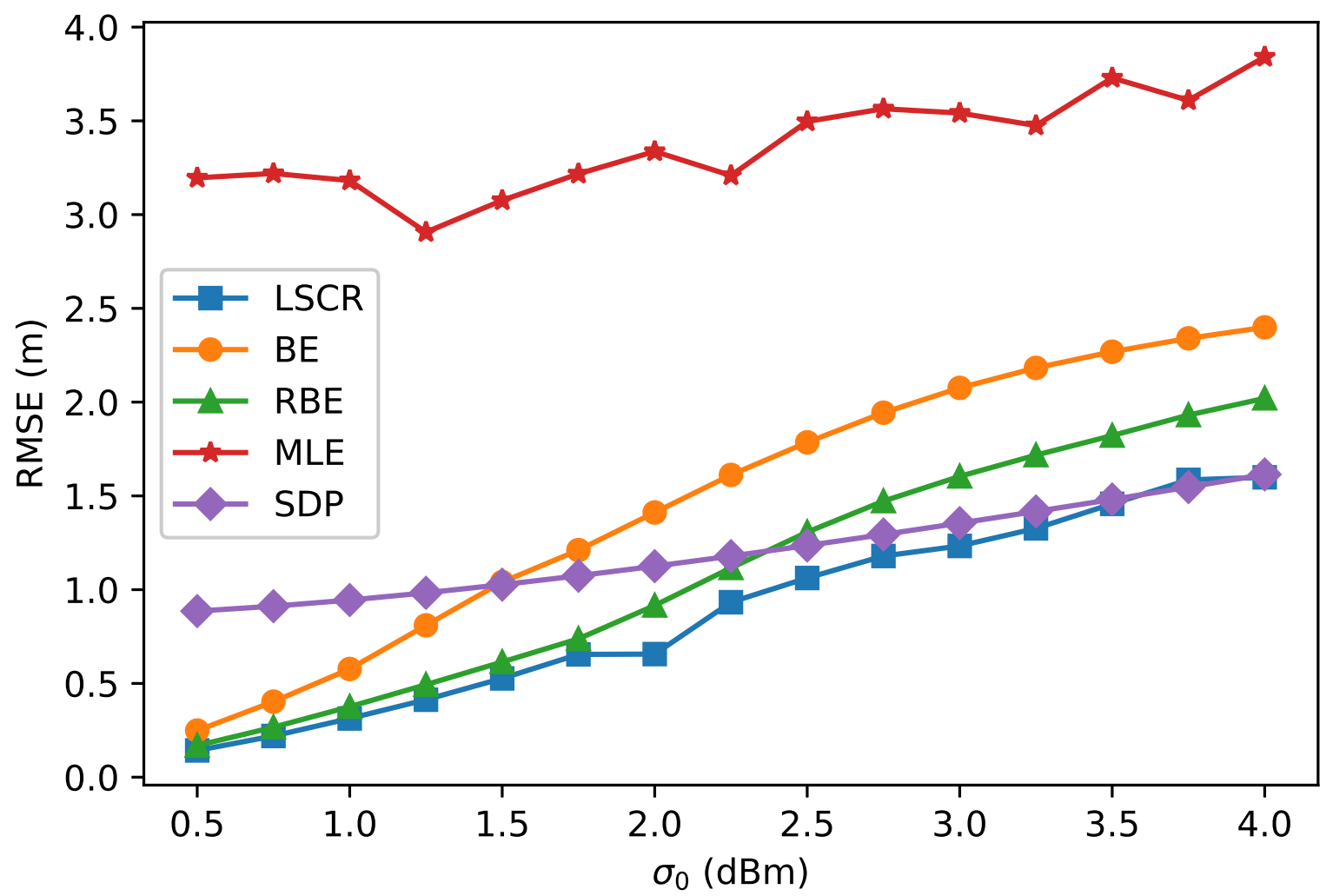

Fig. 4. Evolution of the RMS localization error as a function of $\sigma_{0}$

Figure 5 shows that the proportions of cases the inner and outer approximations of the confidence region as defined by the LSCR approach actually contain the true value of the location are enclosing the targeted $90 \%$ level. In the case of $\mathrm{BE}$ and $\mathrm{RBE}$, the actual location is contained in more cases, but the confidence level is more difficult to predict. The confidence regions associated to the estimates provided by the MLE and SDP approaches are often too optimistic.

Figure 6 shows the average surface of the projection on the $\left(\theta_{1}, \theta_{2}\right)$-plane of the NACR, of the BE and RBE set estimates, and of the confidence region derived from the CRLB. The size of the confidence regions provided by the CRLB (MLE and SDP approaches) are the smallest, but are not consistent with the quality of the provided estimate. The LSCR and RBE approaches provide sets of similar size, while the size of the set provided by the BE approach is larger due to the larger noise bounds considered. 

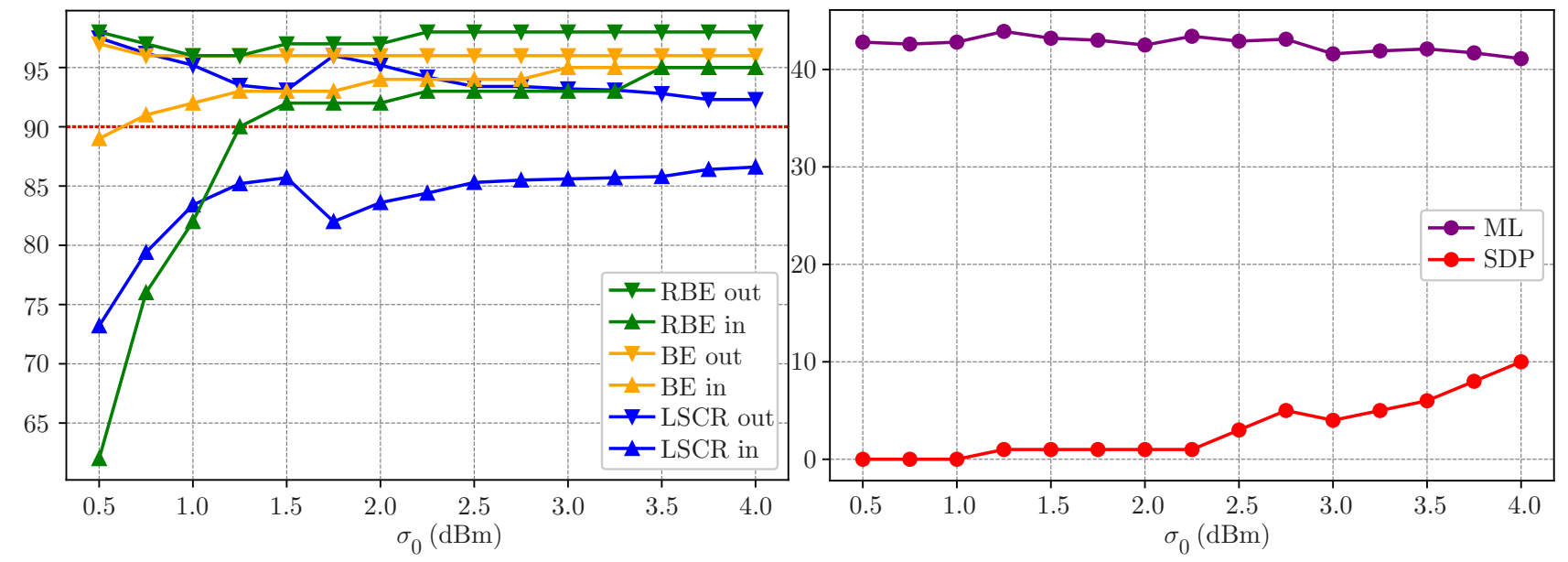

Fig. 5. Proportions of realizations for which the true value of the agent location is contained in the $90 \%$ NACR (left), in the BE set estimates (left), or in the $90 \%$ confidence regions derived from the CRLB (right)

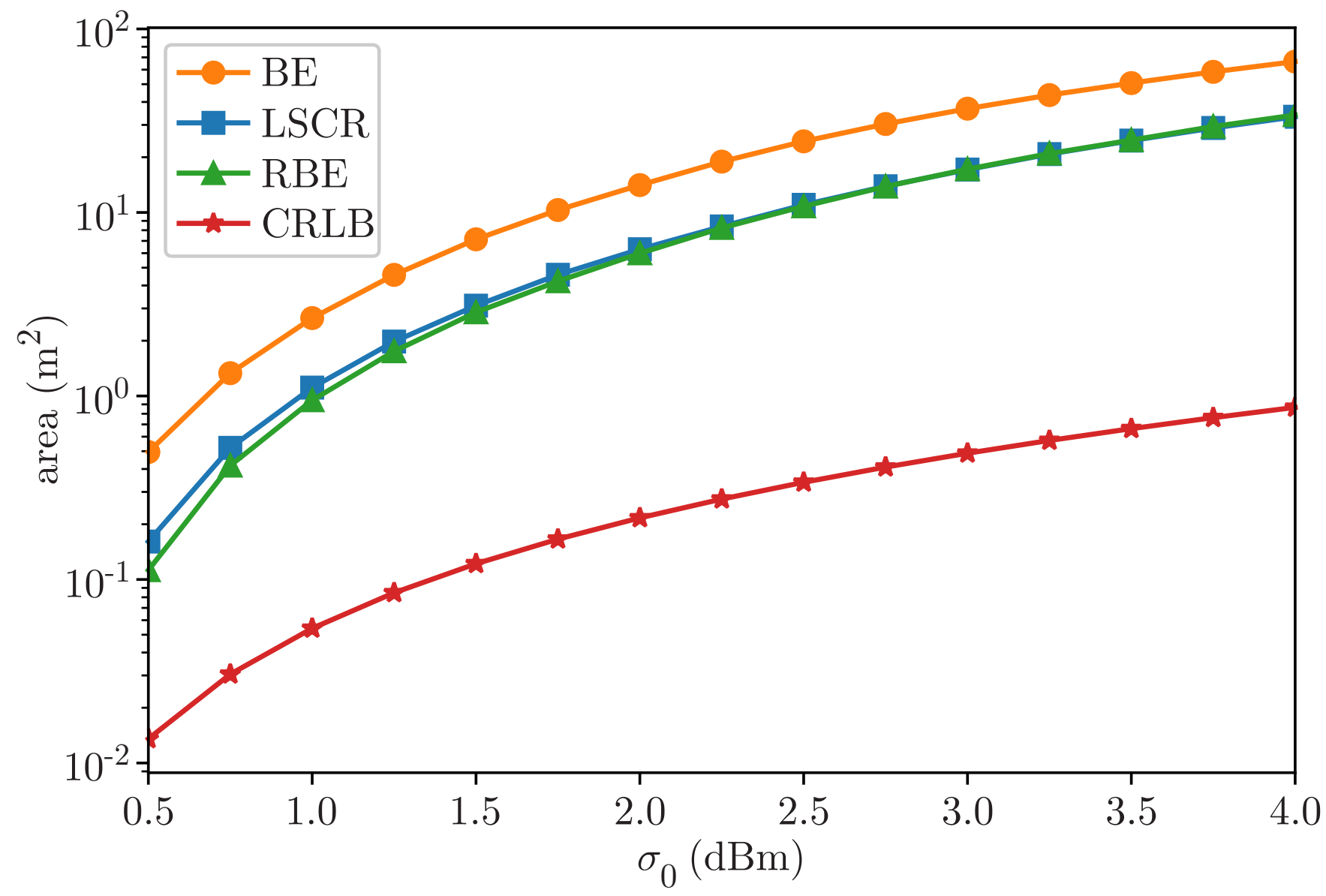

Fig. 6. Evolution of the average surface of the projection on the $\left(\theta_{1}, \theta_{2}\right)$-plane of the NACRs, the set estimates, or the confidence region derived from the CRLB 


\section{Conclusions}

This paper shows that NACR may be obtained using the LSCR approach applied to the problem of source localization from RSS measurements. Inner and outer-approximations of the NACR are provided using tools from interval analysis. Contrary to confidence regions provided by CRLB associated to MLE or SDP techniques, NACR provided by LSCR contains the true value of the parameter vector to be estimated with the prescribed confidence level. Furthermore, LSCR provides the best localization result: its average error is smaller than other compared methods (BE, RBE, MLE, SDP).

The characterization of NACR using interval analysis is for the time being relatively time consuming. More efficient contractors are currently searched to speed-up the characterization process. Applications of the LSCR to recursive estimation is also considered.

\section{A Choice of e for the LSCR approach}

In this appendix, one analyzes the behavior of $s_{j, \mathbf{e}}(\mathbf{p}), j=1, \ldots, m$ when $n_{\mathrm{y}}$ tends to infinity, assuming that $n_{\mathrm{a}}$ anchor nodes provide each the same number $n$ of measurements tending to infinity. The parameters $m$ and $q$ are fixed. Our aim is to show that for properly chosen vectors $\mathbf{e}$, when $\mathbf{p} \neq \mathbf{p}^{*}$, the functions $s_{j, \mathbf{e}}(\mathbf{p}), j=1, \ldots, m$ tend to have all the same sign as $n_{\mathrm{y}}$ tends to infinity. Moreover, to ensure that the NACR shrinks toward $\mathbf{p}^{*}$, one has to determine in which cases, $\mathbf{p}=\mathbf{p}^{*}$ is the unique solution of the equations $s_{j, \mathbf{e}}(\mathbf{p})=0, j=1, \ldots, m$. To perform this analysis, one assumes that the $w_{j}$ are independent and identically distributed.

\section{A.1 First organization of the vector of measurements}

One considers first an organization of the measurement vector as described in (6) and $\mathbf{e}=(1,1)^{T}$ (thus $s=1$ ). Moreover, the groups $\mathbb{G}^{n_{y}-s}$ are assumed to be constructed as described in Apprendix (B). For a given $j$, one considers the elements $\mathbb{I}_{j}^{n_{\mathrm{y}}-s}$ of $\mathbb{G}^{n_{\mathrm{y}}-s}$ with increasing $n_{\mathrm{y}}$ and focus on values of $n_{\mathrm{y}}-s=k n_{\mathrm{a}}(m-1)$, with $k=1, \ldots$ In that case, for a given $j$, one gets

$$
s_{j, \mathbf{e}}(\mathbf{p})=\frac{1}{\left|\mathbb{I}_{j}^{k n_{\mathrm{a}}(m-1)}\right|} \sum_{\ell \in \mathbb{I}_{j}^{k n_{\mathrm{a}}(m-1)}} c_{\ell, \mathbf{e}}(\mathbf{p}),
$$

where, according to the way $\mathbb{G}^{n_{\mathrm{y}}-s}$ has been constructed, $\mathbb{I}_{j}^{k n_{\mathrm{a}}(m-1)}$ contains sequences of $k n_{\mathrm{a}}$ consecutive indices. 
With $\mathbf{e}=(1,1)^{T}$, one has

$$
c_{t,(1,1)}(\mathbf{p})=w_{t}(\mathbf{p}) w_{t+1}(\mathbf{p}),
$$

which, with the measurement vector (6), may take two forms, depending on whether $w_{t}(\mathbf{p})$ and $w_{t+1}(\mathbf{p})$ involve measurements from the same anchor node (most of the cases), or from different anchor nodes, which is the case when $t \in\left\{n, 2 n, \ldots,\left(n_{\mathrm{a}}-1\right) n\right\}$. In the first case

$$
\begin{aligned}
c_{t,(1,1)}(\mathbf{p}) & =\left(\left(P_{0}^{*}-10 \gamma_{\mathrm{P}}^{*} \log _{10} \frac{\left\|\boldsymbol{\theta}_{0}^{*}-\boldsymbol{\theta}_{i_{t}}\right\|}{d_{0}}+w_{t}\right)-\left(P_{0}^{*}-10 \gamma_{\mathrm{P}}^{*} \log _{10} \frac{\left\|\boldsymbol{\theta}_{0}-\boldsymbol{\theta}_{i_{t}}\right\|}{d_{0}}\right)\right) \\
& \left(\left(P_{0}^{*}-10 \gamma_{\mathrm{P}}^{*} \log _{10} \frac{\left\|\boldsymbol{\theta}_{0}^{*}-\boldsymbol{\theta}_{i_{\ell}}\right\|}{d_{0}}+w_{t+1}\right)-\left(P_{0}^{*}-10 \gamma_{\mathrm{P}}^{*} \log _{10} \frac{\left\|\boldsymbol{\theta}_{0}-\boldsymbol{\theta}_{i_{\ell}}\right\|}{d_{0}}\right)\right) \\
& =\left(10 \gamma_{\mathrm{P}}^{*} \log _{10} \frac{\left\|\boldsymbol{\theta}_{0}-\boldsymbol{\theta}_{i_{t}}\right\|}{\left\|\boldsymbol{\theta}_{0}^{*}-\boldsymbol{\theta}_{i_{t}}\right\|}+w_{t}\right)\left(10 \gamma_{\mathrm{P}}^{*} \log _{10} \frac{\left\|\boldsymbol{\theta}_{0}-\boldsymbol{\theta}_{i_{t}}\right\|}{\left\|\boldsymbol{\theta}_{0}^{*}-\boldsymbol{\theta}_{i_{t}}\right\|}+w_{t+1}\right),
\end{aligned}
$$

where $i_{t}$ is the index of the anchor node which has produced measurement $t$. Similarly, in the second case,

$$
c_{t,(1,1)}(\mathbf{p})=\left(10 \gamma_{\mathrm{P}}^{*} \log _{10} \frac{\left\|\boldsymbol{\theta}_{0}-\boldsymbol{\theta}_{i_{t}}\right\|}{\left\|\boldsymbol{\theta}_{0}^{*}-\boldsymbol{\theta}_{i_{t}}\right\|}+w_{t}\right)\left(10 \gamma_{\mathrm{P}}^{*} \log _{10} \frac{\left\|\boldsymbol{\theta}_{0}-\boldsymbol{\theta}_{i_{t}+1}\right\|}{\left\|\boldsymbol{\theta}_{0}^{*}-\boldsymbol{\theta}_{i_{t}+1}\right\|}+w_{t+1}\right) .
$$

One may rewrite (A.1) as

$$
s_{j, \mathbf{e}}(\mathbf{p})=s_{j, \mathbf{e}}^{(1)}(\mathbf{p})+s_{j, \mathbf{e}}^{(2)}(\mathbf{p})
$$

with

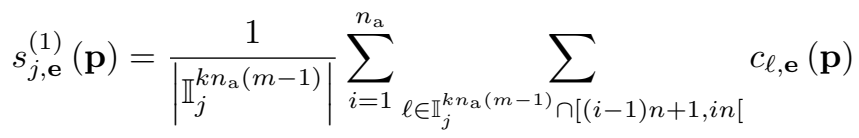

and

$$
\begin{aligned}
s_{j, \mathbf{e}}^{(2)}(\mathbf{p}) & =\frac{1}{\left|\mathbb{I}_{j}^{k n_{\mathrm{a}}(m-1)}\right|} \sum_{\ell \in \mathbb{I}_{j}^{k n_{\mathrm{a}}(m-1)} \cap\left\{n, \ldots,\left(n_{\mathrm{a}}-1\right) n\right\}} c_{\ell, \mathbf{e}}(\mathbf{p}) \\
& =\frac{1}{k\left|\mathbb{I}_{j}^{n_{\mathrm{a}}(m-1)}\right|} \sum_{\ell \in \mathbb{I}_{j}^{k n_{\mathrm{a}}(m-1)} \cap\left\{n, \ldots,\left(n_{\mathrm{a}}-1\right) n\right\}} c_{\ell, \mathbf{e}}(\mathbf{p}) .
\end{aligned}
$$

In $s_{j, \mathbf{e}}^{(1)}(\mathbf{p})$, the second sum with indices $\ell \in \mathbb{I}_{j}^{k n_{\mathrm{a}}(m-1)} \cap\left[(i-1) n+1, i n\left[\right.\right.$ considers only the $c_{\ell, \mathbf{e}}(\mathbf{p})$ involving measurements from the same anchor node $i$. In $s_{j, \mathbf{e}}^{(2)}(\mathbf{p})$, the sum with indices $\ell \in \mathbb{I}_{j}^{k n_{\mathrm{a}}(m-1)} \cap\left\{n, \ldots,\left(n_{\mathrm{a}}-1\right) n\right\}$ considers only the $c_{\ell, \mathbf{e}}(\mathbf{p})$ involving measurements from different anchor nodes.

Since $\mathbb{I}_{j}^{k n_{\mathrm{a}}(m-1)}$ contains sequences of $k n_{\mathrm{a}}$ consecutive indices and $\mathbb{I}_{j}^{n_{\mathrm{a}}(m-1)}$ contains sequences of $n_{\mathrm{a}}$ consecutive indices, using $\mathbb{I}_{j}^{n_{\mathrm{a}}(m-1)}$ one may deduce the indices present in $\mathbb{I}_{j}^{k n_{\mathrm{a}}(m-1)}$. For example, if $\ell=1 \in \mathbb{I}_{j}^{n_{\mathrm{a}}(m-1)}$, then 
$\{1, \ldots, k\} \subset \mathbb{I}_{j}^{k n_{\mathrm{a}}(m-1)}$. More generally, if $\ell \in \mathbb{I}_{j}^{n_{\mathrm{a}}(m-1)}$, then $\{(\ell-1) k+1, \ldots, \ell k\} \subset \mathbb{I}_{j}^{k n_{\mathrm{a}}(m-1)}$. Consequently, (A.5) may be rewritten as

$$
\begin{aligned}
s_{j, \mathbf{e}}^{(1)}(\mathbf{p}) & =\frac{1}{\left|\mathbb{I}_{j}^{k n_{\mathrm{a}}(m-1)}\right|} \sum_{i=1}^{n_{\mathrm{a}}} \sum_{\kappa=1}^{k} \sum_{\ell \in \mathbb{I}_{j}^{n_{\mathrm{a}}(m-1)},(i-1) n+1 \leqslant(\ell-1) k+\kappa<i n} c_{(\ell-1) k+\kappa, \mathbf{e}}(\mathbf{p}) \\
& =\frac{1}{k \mid \mathbb{I}_{j}^{n_{\mathrm{a}}(m-1) \mid}} \sum_{i=1}^{n_{\mathrm{a}}} \sum_{\ell \in \mathbb{I}_{j}^{n_{\mathrm{a}}(m-1)}} \sum_{\kappa=1,(i-1) n+1 \leqslant(\ell-1) k+\kappa<i n} c_{(\ell-1) k+\kappa, \mathbf{e}}(\mathbf{p}) \\
& =\frac{1}{\mid \mathbb{I}_{j}^{n_{\mathrm{a}}(m-1) \mid}} \sum_{i=1}^{n_{\mathrm{a}}} \sum_{\ell \in \mathbb{I}_{j}^{n_{\mathrm{a}}(m-1)}} \frac{1}{k} \sum_{\kappa=1,(i-1) n+1 \leqslant(\ell-1) k+\kappa<i n}^{k} c_{(\ell-1) k+\kappa, \mathbf{e}}(\mathbf{p}) .
\end{aligned}
$$

Then, using similar arguments as those considered in (7, Appendix A.2), for all $\mathbf{p}$ such that the associated $\boldsymbol{\theta}_{0} \neq \boldsymbol{\theta}_{i}$, $i=1, \ldots, n_{\mathrm{a}}$, in the case $\mathbf{e}=(1,1)^{T}$, one has

$$
\lim _{k \rightarrow \infty} \frac{1}{k} \sum_{\kappa=1,(i-1) n+1 \leqslant(\ell-1) k+\kappa<i n}^{k} c_{(\ell-1) k+\kappa,(1,1)}(\mathbf{p})=\left(10 \gamma_{\mathrm{P}}^{*} \log _{10} \frac{\left\|\boldsymbol{\theta}_{0}-\boldsymbol{\theta}_{i}\right\|}{\left\|\boldsymbol{\theta}_{0}^{*}-\boldsymbol{\theta}_{i}\right\|}\right)^{2}
$$

which is the expected value of $c_{t,(1,1)}(\mathbf{p})$ for some $t$ such that $i_{t}=i$.

Moreover, since $s_{j, \mathbf{e}}^{(2)}(\mathbf{p})$ consists only in $n_{\mathrm{a}}$ terms, for all $\mathbf{p}$ such that the associated $\boldsymbol{\theta}_{0} \neq \boldsymbol{\theta}_{i}, i=1, \ldots, n_{\mathrm{a}}$, one has

$$
\lim _{k \rightarrow \infty} \frac{1}{k\left|\mathbb{I}_{j}^{n_{\mathrm{a}}(m-1) \mid}\right|} \sum_{\ell \in \mathbb{I}_{j}^{k n_{\mathrm{a}}(m-1)} \cap\left\{n, \ldots,\left(n_{\mathrm{a}}-1\right) n\right\}} c_{\ell, \mathbf{e}}(\mathbf{p})=0
$$

Let $\alpha_{\ell, i} \geqslant 0$ be the proportion of indices in $\mathbb{I}_{\ell}^{n_{\mathrm{y}}-s}$ corresponding to $c_{t,(1,1)}(\mathbf{p})$ involving measurements of anchor node $i$ only. When $n_{\mathrm{y}}-s=k n_{\mathrm{a}}(m-1)$, these proportions are independent of $k$. As a consequence, for all $\mathbf{p}$ such that the associated $\boldsymbol{\theta}_{0} \neq \boldsymbol{\theta}_{i}, i=1, \ldots, n_{\mathrm{a}}$, one has

$$
\begin{aligned}
s_{j,(1,1)}^{\infty}(\mathbf{p}) & =\lim _{k \rightarrow \infty} s_{j,(1,1)}(\mathbf{p}) \\
& =\sum_{i=1}^{n_{\mathrm{a}}} \alpha_{\ell, i}\left(10 \gamma_{\mathrm{P}}^{*} \log _{10} \frac{\left\|\boldsymbol{\theta}_{0}-\boldsymbol{\theta}_{i}\right\|}{\left\|\boldsymbol{\theta}_{0}^{*}-\boldsymbol{\theta}_{i}\right\|}\right)^{2}
\end{aligned}
$$

which vanishes for all $\boldsymbol{\theta}_{0}$ such that

$$
\left\|\boldsymbol{\theta}_{0}-\boldsymbol{\theta}_{i}\right\|=\left\|\boldsymbol{\theta}_{0}^{*}-\boldsymbol{\theta}_{i}\right\|, i \in\left\{1, \ldots, n_{\mathrm{a}} \mid \alpha_{\ell, i}>0\right\} .
$$

Provided that for each $\ell$ there are at least three non-aligned anchor nodes such that $\alpha_{\ell, i}>0, \boldsymbol{\theta}_{0}=\boldsymbol{\theta}_{0}^{*}$ is the unique solution of the system of equations (A.7). Any $\boldsymbol{\theta}_{0} \neq \boldsymbol{\theta}_{0}^{*}$ leads to strictly positive $s_{j,(1,1)}^{\infty}(\mathbf{p})$. 
In the case $\mathbf{e}=(3,1)^{T}$, using similar derivations, one obtains

$$
s_{j,(3,1)}^{\infty}(\mathbf{p})=\sum_{i=1}^{n_{\mathrm{a}}} \alpha_{\ell, i}\left(10 \gamma_{\mathrm{P}}^{*} \log _{10} \frac{\left\|\boldsymbol{\theta}_{0}-\boldsymbol{\theta}_{i}\right\|}{\left\|\boldsymbol{\theta}_{0}^{*}-\boldsymbol{\theta}_{i}\right\|}\right)^{4},
$$

and again, $\boldsymbol{\theta}_{0}=\boldsymbol{\theta}_{0}^{*}$ is the unique solution of the system of equations (A.7).

In the case $\mathbf{e}=(2,1)^{T}$, one obtains now

$$
s_{j,(2,1)}^{\infty}(\mathbf{p})=\sum_{i=1}^{n_{\mathrm{a}}} \alpha_{\ell, i}\left(10 \gamma_{\mathrm{P}}^{*} \log _{10} \frac{\left\|\boldsymbol{\theta}_{0}-\boldsymbol{\theta}_{i}\right\|}{\left\|\boldsymbol{\theta}_{0}^{*}-\boldsymbol{\theta}_{i}\right\|}\right)^{3},
$$

which when $\boldsymbol{\theta}_{0}$ is close to $\boldsymbol{\theta}_{0}^{*}$ but $\boldsymbol{\theta}_{0} \neq \boldsymbol{\theta}_{0}^{*}$ depends on the values of $\alpha_{\ell, i}, i=1, \ldots, n_{\mathrm{a}}$. For this choice of e, the NACR will not shrink to $\mathbf{p}^{*}$ when $n_{\mathrm{y}}$ tends to infinity.

When both $\boldsymbol{\theta}_{0}$ and $P_{0}$ have to be estimated, in the case $\mathbf{e}=(1,1)^{T}$, one obtains

$$
\begin{aligned}
c_{t,(1,1)}(\mathbf{p}) & =\left(\left(P_{0}^{*}-10 \gamma_{\mathrm{P}}^{*} \log _{10} \frac{\left\|\boldsymbol{\theta}_{0}^{*}-\boldsymbol{\theta}_{i_{t}}\right\|}{d_{0}}+w_{t}\right)-\left(P_{0}-10 \gamma_{\mathrm{P}}^{*} \log _{10} \frac{\left\|\boldsymbol{\theta}_{0}-\boldsymbol{\theta}_{i_{t}}\right\|}{d_{0}}\right)\right) \\
& \left(\left(P_{0}^{*}-10 \gamma_{\mathrm{P}}^{*} \log _{10} \frac{\left\|\boldsymbol{\theta}_{0}^{*}-\boldsymbol{\theta}_{i_{\ell}}\right\|}{d_{0}}+w_{t+1}\right)-\left(P_{0}-10 \gamma_{\mathrm{P}}^{*} \log _{10} \frac{\left\|\boldsymbol{\theta}_{0}-\boldsymbol{\theta}_{i_{\ell}}\right\|}{d_{0}}\right)\right) \\
& =\left(P_{0}^{*}-P_{0}+10 \gamma_{\mathrm{P}}^{*} \log _{10} \frac{\left\|\boldsymbol{\theta}_{0}-\boldsymbol{\theta}_{i_{t}}\right\|}{\left\|\boldsymbol{\theta}_{0}^{*}-\boldsymbol{\theta}_{i_{t}}\right\|}+w_{t}\right)\left(P_{0}^{*}-P_{0}+10 \gamma_{\mathrm{P}}^{*} \log _{10} \frac{\left\|\boldsymbol{\theta}_{0}-\boldsymbol{\theta}_{i_{t}}\right\|}{\left\|\boldsymbol{\theta}_{0}^{*}-\boldsymbol{\theta}_{i_{t}}\right\|}+w_{t+1}\right),
\end{aligned}
$$

when measurements from the same sensors are combined. Then one gets

$$
s_{j,(1,1)}^{\infty}(\mathbf{p})=\sum_{i=1}^{n_{\mathrm{a}}} \alpha_{\ell, i}\left(P_{0}^{*}-P_{0}+10 \gamma_{\mathrm{P}}^{*} \log _{10} \frac{\left\|\boldsymbol{\theta}_{0}-\boldsymbol{\theta}_{i}\right\|}{\left\|\boldsymbol{\theta}_{0}^{*}-\boldsymbol{\theta}_{i}\right\|}\right)^{2},
$$

which vanishes for all $\left(P_{0}, \boldsymbol{\theta}_{0}\right)$ such that

$$
P_{0}^{*}-P_{0}+10 \gamma_{\mathrm{P}}^{*} \log _{10} \frac{\left\|\boldsymbol{\theta}_{0}-\boldsymbol{\theta}_{i}\right\|}{\left\|\boldsymbol{\theta}_{0}^{*}-\boldsymbol{\theta}_{i}\right\|}=0, i=1, \ldots, n_{\mathrm{a}} .
$$

This condition may be rewritten as

$$
\left\|\boldsymbol{\theta}_{0}-\boldsymbol{\theta}_{i}\right\|=\left\|\boldsymbol{\theta}_{0}^{*}-\boldsymbol{\theta}_{i}\right\| \times 10^{\frac{P_{0}-P_{0}^{*}}{10 \gamma_{\mathrm{p}}^{*}}}, i=1, \ldots, n_{\mathrm{a}} .
$$

If $P_{0}$ is known to belong to some a priori search interval $\left[\underline{P}_{0}, \bar{P}_{0}\right]$, then $\boldsymbol{\theta}_{0}$ has to belong to the intersection of annulus centered in $\boldsymbol{\theta}_{i}$ with radius between $\left\|\boldsymbol{\theta}_{0}^{*}-\boldsymbol{\theta}_{i}\right\| \times 10^{\frac{P_{0}-P_{0}^{*}}{10 \gamma_{\mathrm{p}}^{*}}}$ and $\left\|\boldsymbol{\theta}_{0}^{*}-\boldsymbol{\theta}_{i}\right\| \times 10^{\frac{\bar{P}_{0}-P_{*}^{*}}{10 \gamma_{\mathrm{p}}^{*}}}$. The intersection of these annuli defines the NACR for $\boldsymbol{\theta}_{0}$, and may also lead to a reduced confidence region for $P_{0}$. Nevertheless, in this case, the confidence region does not shrink to a single point in the search space.

Similar conclusions can be obtained when both $\boldsymbol{\theta}_{0}$ and $\gamma_{\mathrm{P}}$ or $\boldsymbol{\theta}_{0}, P_{0}$, and $\gamma_{\mathrm{P}}$ have to be estimated. 


\section{A.2 Alternative organization of the vector of measurements}

Consider now an organization of the measurement vector as described in (36) and $\mathbf{e}=(1,1)^{T}$. The expression of $c_{t, \mathbf{e}}(\mathbf{p})$ is still given by (A.2), but now, all $c_{t, \mathbf{e}}(\mathbf{p})$ will involve measurements from two different anchor nodes. After some derivations, one obtains now

$$
\begin{aligned}
s_{j,(1,1)}^{\infty}(\mathbf{p}) & =\sum_{i=1}^{n_{\mathrm{a}}-1} \beta_{\ell, i}\left(10 \gamma_{\mathrm{P}}^{*} \log _{10} \frac{\left\|\boldsymbol{\theta}_{0}-\boldsymbol{\theta}_{i}\right\|}{\left\|\boldsymbol{\theta}_{0}^{*}-\boldsymbol{\theta}_{i}\right\|}\right)\left(10 \gamma_{\mathrm{P}}^{*} \log _{10} \frac{\left\|\boldsymbol{\theta}_{0}-\boldsymbol{\theta}_{i+1}\right\|}{\left\|\boldsymbol{\theta}_{0}^{*}-\boldsymbol{\theta}_{i+1}\right\|}\right) \\
& +\beta_{\ell, n_{\mathrm{a}}}\left(10 \gamma_{\mathrm{P}}^{*} \log _{10} \frac{\left\|\boldsymbol{\theta}_{0}-\boldsymbol{\theta}_{n_{\mathrm{a}}}\right\|}{\left\|\boldsymbol{\theta}_{0}^{*}-\boldsymbol{\theta}_{n_{\mathrm{a}}}\right\|}\right)\left(10 \gamma_{\mathrm{P}}^{*} \log _{10} \frac{\left\|\boldsymbol{\theta}_{0}-\boldsymbol{\theta}_{1}\right\|}{\left\|\boldsymbol{\theta}_{0}^{*}-\boldsymbol{\theta}_{1}\right\|}\right)
\end{aligned}
$$

where $\beta_{\ell, i}$ is the proportion in $s_{j,(1,1)}(\mathbf{p})$ of terms $c_{t, \mathbf{e}}(\mathbf{p})$ involving a measurement provided by the $i$-th and the $i+1 \bmod n_{\mathrm{a}}$ anchor nodes. In that case, $s_{j,(1,1)}^{\infty}(\mathbf{p})$ is positive for values of $\boldsymbol{\theta}_{0}$ far avay from the anchor nodes, i.e., such that $\left\|\boldsymbol{\theta}_{0}-\boldsymbol{\theta}_{i}\right\|>\left\|\boldsymbol{\theta}_{0}^{*}-\boldsymbol{\theta}_{i}\right\|$ for all $i=1, \ldots, n_{a}$. When $\boldsymbol{\theta}_{0}$ is close to $\boldsymbol{\theta}_{0}^{*}$ the sign of $s_{j,(1,1)}^{\infty}(\mathbf{p})$ is difficult to predict and unlikely to be the same for all $s_{j,(1,1)}^{\infty}(\mathbf{p}), j=1, \ldots, m$.

\section{B Construction of the groups $\mathbb{G}^{N}$}

The analysis of the behavior of the LSCR approach in the considered case where different measurement models have to be considered requires a slight adaptation of the construction of the groups $\mathbb{G}^{N}$ used by the LSCR approach as presented in (7, Appendix A.3).

Consider the set $\mathbb{I}^{N}=\{1, \ldots, N\}$ and an integer $m=2^{\mu}$. To build a group $\mathbb{G}^{N}$ for the symmetric difference operator, as in (7, Appendix A.3), one considers first Gordon's iterative construction (10). One starts with $\mathbf{R}(1)=[1]$ and evaluate

$$
\mathbf{R}(k)=\left[\begin{array}{ccc}
\mathbf{R}(k-1) & \mathbf{R}(k-1) & \mathbf{0} \\
\mathbf{R}(k-1) & \mathbf{J}-\mathbf{R}(k-1) & \mathbf{1} \\
\mathbf{0}^{T} & \mathbf{1}^{T} & 1
\end{array}\right], k=1, \ldots, \mu,
$$

where $\mathbf{J}$ is the all-one matrix of appropriate size, and $\mathbf{0}$ and $\mathbf{1}$ are respectively the all-zero and all-one vectors of appropriate size. Then, one considers

$$
\mathbf{Q}^{m-1}=\left[\begin{array}{c}
\mathbf{R}(k) \\
\mathbf{0}^{T}
\end{array}\right]=\left[\mathbf{q}_{1}, \ldots, \mathbf{q}_{m-1}\right]
$$


where the $\mathbf{q}_{i}$ s are the column vectors of $\mathbf{Q}^{m-1}$. Then, one build the matrix

$$
\mathbf{Q}=\left[\mathbf{q}_{1}, \ldots, \mathbf{q}_{1}, \mathbf{q}_{2}, \ldots, \mathbf{q}_{2}, \ldots, \mathbf{q}_{m-1}, \ldots, \mathbf{q}_{m-1}\right]
$$

where each column vector is repeated $\lceil N /(m-1)\rceil$ times so that $\mathbf{Q}$ has at least $N$ columns. The matrix $\mathbf{G}$ obtained selecting the first $N$ columns of $\mathbf{Q}$ is the incidence matrix of the group $\mathbb{G}^{N}$. $\mathbf{Q}$ is such that $Q_{i, j}=1$ if $j \in \mathbb{I}_{i}^{N}$.

\section{References}

[1] M. C. Campi and E. Weyer. Guaranteed non-asymptotic confidence regions in system identification. Automatica, 41(10):1751-1764, 2005.

[2] M. C. Campi and E. Weyer. Non-asymptotic confidence sets for input-output transfer functions. In Decision and Control, pages 157-162, 2006.

[3] M. C. Campi and E. Weyer. Non-asymptotic confidence sets for the parameters of linear transfer functions. IEEE Transactions on Automatic Controls, 55(12):2708-2720, 2010.

[4] G. Chabert and L. Jaulin. Contractor programming. Artificial Intelligence, 173(11):1079-1100, 2009.

[5] B. C. Csáji, M. C. Campi, and E. Weyer. Non-asymptotic confidence regions for the least-squares estimate. In IFAC Symposium on System Identification, pages 227-232, Brussels, Belgium, 2012.

[6] B. C. Csáji, M. C. Campi, and E. Weyer. Sign-perturbed sums: A new system identification approach for constructing exact non-asymptotic confidence regions in linear regression models. IEEE Transactions on Signal Processing, 63(1):169-181, Jan 2015.

[7] M. Dalai, E. Weyer, and M. C. Campi. Parameter identification for nonlinear systems: Guaranteed confidence regions through LSCR. Automatica, 43:1418 - 1425, 2007.

[8] S. Gezici. A survey on wireless position estimation. Wireless personal communications, 44(3):263-282, 2008.

[9] M. R. Gholami, H. Wymeersch, E. G. Ström, and M. Rydström. Wireless network positioning as a convex feasibility problem. Wireless Communications and Networking, 2011(1):1-15, 2011.

[10] L. Gordon. Completely separating groups in subsampling. Annals of Statistics, 2(3):572-578, 1974.

[11] N. Iliev and I. Paprotny. Review and comparison of spatial localization methods for low-power wireless sensor networks. IEEE Sensors Journal, 15(10):5971-5987, 2015.

[12] L. Jaulin, M. Kieffer, O. Didrit, and E. Walter. Applied interval analysis: with examples in parameter and state estimation, robust control and robotics, volume 1. Springer Science \& Business Media, 2001. 
[13] L. Jaulin and E. Walter. Guaranteed nonlinear parameter estimation from bounded-error data via interval analysis. Mathematics and Computers in Simulation, 35(2):123-137, 1993.

[14] L. Jaulin and E. Walter. Set inversion via interval analysis for nonlinear bounded-error estimation. Automatica, 29(4):1053-1064, 1993.

[15] L. Jaulin, E. Walter, and O. Didrit. Guaranteed robust nonlinear parameter bounding. In Computational Engineering in Systems Applications, 1996.

[16] M. Kieffer and E. Walter. Guaranteed characterization of exact non-asymptotic confidence regions as defined by LSCR and SPS. Automatica, 50(2):507-512, 2014.

[17] S. Kim, H. Jeon, and J. Ma. Robust localization with unknown transmission power for cognitive radio. In Military Communications Conference, pages 1-6. IEEE, 2007.

[18] J. H. Lee and R. M. Buehrer. Location estimation using differential RSS with spatially correlated shadowing. In IEEE International Conference on Global Telecommunications, 2009.

[19] J. Léger and M. Kieffer. Guaranteed robust distributed estimation in a network of sensors. In IEEE International Conference on Acoustics Speech and Signal Processing, 2010.

[20] X. Li, Z. D. Deng, L. T. Rauchenstein, and T. J. Carlson. Contributed review: Source-localization algorithms and applications using time of arrival and time difference of arrival measurements. Review of Scientific Instruments, $87(4): 041502,2016$.

[21] H. Lohrasbipeydeh, T. A. Gulliver, and H. Amindavar. A minimax SDP method for energy based source localization with unknown transmit power. IEEE Wireless Communications Letters, 3(4):433-436, 2014.

[22] M. Milanese, J. Norton, H. Piet-Lahanier, and E. Walter, editors. Bounding Approaches to System Identification. Plenum Press, New York, NY, 1996.

[23] R. E. Moore. Interval analysis. englewood cliff, 1966.

[24] F. Mourad, H. Snoussi, F. Abdallah, and C. Richard. Anchor-based localization via interval analysis for mobile ad-hoc sensor networks. IEEE Transactions on Signal Processing, 57(8):3226-3239, 2009.

[25] F. Mourad, H. Snoussi, M. Kieffer, and C. Richard. Robust interval-based localization algorithms for mobile sensor networks. International Journal of Distributed Sensor Networks, 8(1):303895, 2011.

[26] J. P. Norton, editor. Special Issue on Bounded-Error Estimation: Issue 2. 1995. International Journal of Adaptive Control and Signal Processing 9(1):1-132.

[27] Y. Okumura, E. Ohmori, T. Kawano, and K. Fukuda. Field strength and its variability in VHF and UHF land-mobile radio service. Rev. Elec. Commun. Lab, 16(9):825-73, 1968. 
[28] R. W. Ouyang, A. K. Wong, and C. Lea. Received signal strength-based wireless localization via semidefinite programming: Noncooperative and cooperative schemes. IEEE Transactions on Vehicular Technology, 59(3):1307-1318, 2010.

[29] L. Pronzato and E. Walter. Robustness to outliers of bounded-error estimators and consequences on experiment design. In Bounding Approaches to System Identification, pages 199-212, 1996.

[30] G Rahimi, M. R. Danaee, and S. Bayat. A generalized total least squares algorithm for RSS-based localization with unknown path-loss model parameters. In Iranian Conference on Electrical Engineering, pages 521-524, 2016.

[31] K. C. Toh, M. J. Todd, and R. H. Tütüncü. SDPT3-a matlab software package for semidefinite programming, version 1.3. Optimization methods and software, 11(1-4):545-581, 1999.

[32] S. Tomic, M. Beko, and R. Dinis. RSS-based localization in wireless sensor networks using convex relaxation: Noncooperative and cooperative schemes. IEEE Transactions on Vehicular Technology, 64(5):2037-2050, 2015.

[33] D. J. Torrieri. Statistical theory of passive location systems. In Autonomous robot vehicles, pages 151-166. 1990.

[34] R. M. Vaghefi, M. R. Gholami, R. M. Buehrer, and E. G. Strom. Cooperative received signal strength-based sensor localization with unknown transmit powers. IEEE Transactions on Signal Processing, 61(6):1389-1403, 2013.

[35] G. Wang and K. Yang. Efficient semidefinite relaxation for energy-based source localization in sensor networks. In IEEE International Conference on Acoustics, Speech and Signal Processing, pages 2257-2260, 2009.

[36] J. Wang, Q. Gao, Y. Yu, L. Cheng, P.and Wu, and H. Wang. Robust device-free wireless localization based on differential RSS measurements. IEEE transactions on industrial electronics, 60(12):5943-5952, 2013.

[37] Erik Weyer, Marco Claudio Campi, and Balázs Csanád Csáji. Asymptotic properties of sps confidence regions. Automatica, 82:287 - 294, 2017. 